\title{
A FAMILY OF LINKS AND THE CONWAY CALCULUS
}

\author{
BY
}

COLE A. GILLER

\begin{abstract}
In 1969, J. H. Conway gave efficient methods of calculating abelian invariants of classical knots and links. The present paper includes a detailed exposition (with new proofs) of these methods and extensions in several directions.

The main application given here is as follows. A link $L$ of two unknotted components in $S^{3}$ has the distinct lifting property for $p$ if the lifts of each component to the $p$-fold cover of $S^{3}$ branched along the other are distinct. The $p$-fold covers of these lifts are homeomorphic, and so $L$ gives an example of two distinct knots with the same $p$-fold cover. The above machinery is then used to construct an infinite family of links, each with the distinct lifting property for all $p \geqslant 2$.
\end{abstract}

I. Introduction. Let $L \subseteq S^{3}$ be a link of two unknotted components $L_{1}$ and $L_{2}$, and denote the $p$-fold cover of $S^{3}$ branched along a knot $K$ by $B_{p}(K)$; note that $B_{p}\left(L_{i}\right)$ is homeomorphic to $S^{3}$. We say that $L$ has the distinct lifting property (DLP) for $p$ if the lifts of $L_{1}$ and $L_{2}$ to $B_{p}\left(L_{2}\right)$ and $B_{p}\left(L_{1}\right)$ respectively represent distinct links types in $S^{3}$. Such an $L$ is automatically asymmetric. As we show later, the $p$-fold cover of $S^{3}$ branched along each of these lifts is the same $p^{2}$-fold cover of $S^{3}$ branched along $L$, and so we are provided with a pair of distinct links with homeomorphic $p$-fold covers. It is unknown whether a pair of distinct knots can have homeomorphic $p$-fold covers for all $p$ (see Problem 1.27 in [K]), and we do not know of a pair of knots with homeomorphic $p$-fold covers for two distinct values of $p$.

The purpose of this paper is 2-fold. We first give an example of an infinite family of links $L_{k}, k \geqslant 2$, each with the DLP for $p \geqslant 2$. The associated lifts in each case have identical signature [M2] but are distinguished by their Alexander polynomials. Thus we obtain for each $p$ an infinite family of pairs of distinct links (in fact, knots) with homeomorphic $p$-fold covers.

Our second purpose is to follow J. H. Conway [C1, C2] in outlining a fast and efficient method of calculating abelian invariants of links. In particular, we hope to point to these methods not only as convenient computational tools, but also as a means to prove general facts about links and as being of interest in their own right.

In §II.1 we identify an Alexander type polynomial of an oriented link $L$ as an invariant of a certain infinite cyclic cover of $L$. Specifically, choose an oriented trivial meridian $\mu_{i} \in H_{1}\left(S^{3}-L\right)$ using the right-hand rule for each component of $L$, and let $M_{L}$ be the cover corresponding to the map to $\mathbf{Z}$ which sends each $\mu_{i}$ to a fixed generator $t$. Then $H_{1}\left(M_{L}\right)$ is a $\mathbf{Z}\left[t, t^{-1}\right]$-module in the usual way, and our

Received by the editors February 4, 1980 and, in revised form, July 15, 1980.

1980 Mathematics Subject Classification. Primary 57M25; Secondary 57M12.

(c) 1982 American Mathematical Society 0002-9947/81/0000-1024/\$09.75 
polynomial is the determinant of a presentation matrix for this module. This polynomial is seen to be an isotopy invariant of $L$ but not an invariant of link complement.

In $\$ I I .2$ we give a new proof of the consistency of a method of Conway for the recursive assignment of a polynomial to $L$, and identify this Conway polynomial with the polynomial discussed above. Thus the groundwork is laid for a major theme of this paper: that this recursive machinery can be used to gain information about links without direct recourse to covering spaces of complements. $\$ \S I I .3$ and II.4 begin this theme by giving some computational lemmas and examples useful in the recursion, and by interpreting and reproving some well-known facts about links in our recursive framework. \$II.7 continues by showing how signatures may be computed recursively and by giving a simple algorithm for finding the polynomial of a 2-bridge knot. Our theme finds even more justification in the following manner:

One way to draw the lift of one component $L_{1}$ of a link $L$ to the $p$-fold cover of $S^{3}$ branched along another unknotted component $L_{2}$ is to cut along a disk bounded by $L_{2}$, straighten the strands belonging to $L_{1}$, and juxtapose $p$ of the resulting blocks end-to-end (see Figure 1).
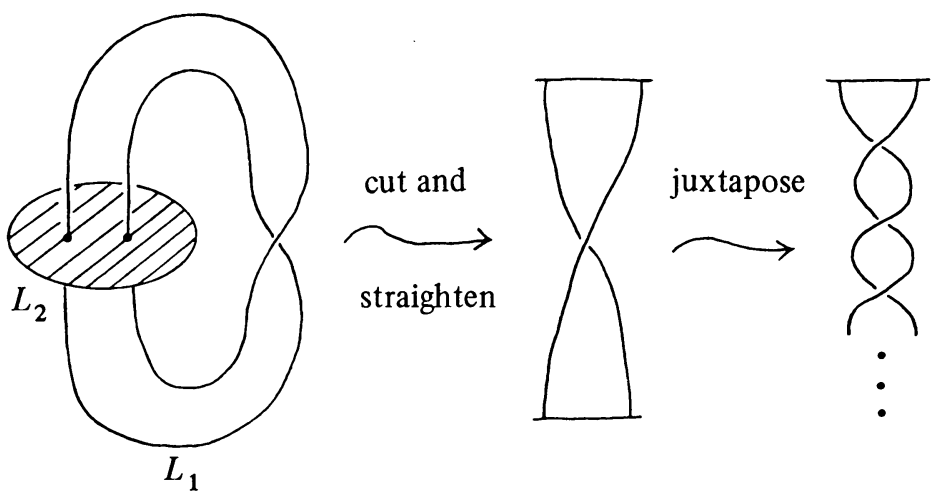

\section{FigURE 1}

In fact, many links can be decomposed into such a block form, and it is desirable to be able to compute polynomials of links in terms of their block components. Following Conway, we define in $\$ I I .5$ an algebra spanned by certain blocks (which Conway calls skein elements) subject to our previous recursion relations. We investigate the structure of this algebra and gain powerful formulas enabling us to perform the above calculation.

After giving examples in §II.6 both for the reader's practice and for our use later in this paper, we define two links to be skein equivalent if they have the same recursive structure, and we define an operation on links called mutation. Again following Conway, we show that mutants are skein equivalent and in this way give an example of a pair of distinct skein equivalent knots; the 2-fold covers of any two mutants are homeomorphic, and we give pictures of the $p$-fold covers of these examples and ask when they are homeomorphic. 
Finally, in §III we give our example of a family of links with the DLP and prove that the distinct lifts have homeomorphic $p$-fold covers. The degree of the polynomial of one of the lifts is calculated recursively by the above methods, although Conway's formula cannot be used directly. The other lift is seen to have genus $\leqslant p$, a fact which prevents the degree of its polynomial from agreeing with that of the other. Thus the two lifts are distinguished by their Conway polynomials.

We use the following conventions. All links are assumed oriented, and arrows indicate the chosen orientation. $n$ stands for $n$ half-twists, right-handed for positive $n$ and left-handed for negative $n$ (for example, t⿹

With these conventions, the family of links mentioned above is

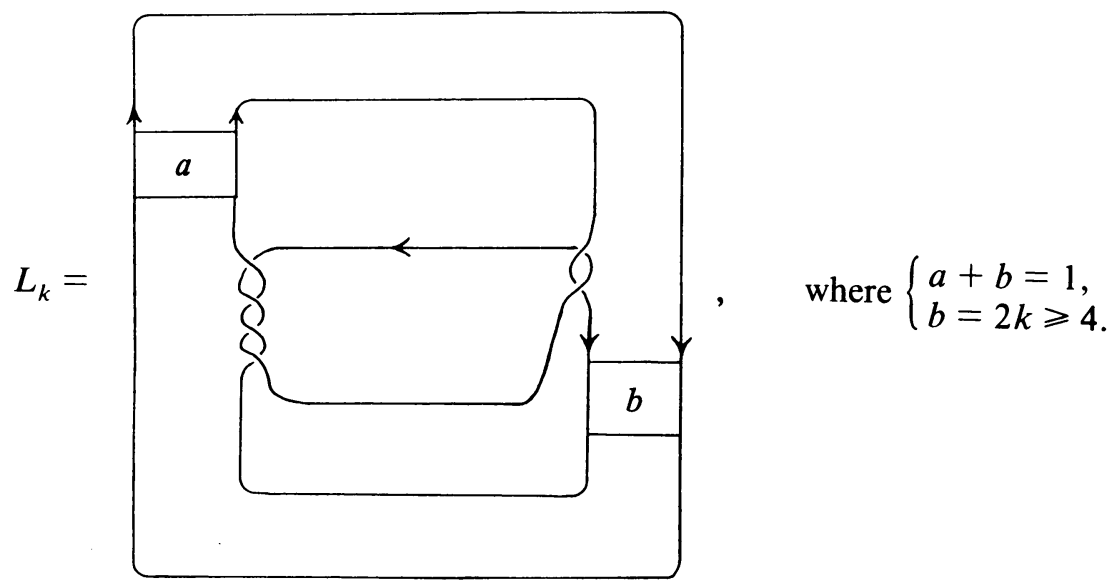

FigURE 2

Note that the linking number of the two components is 1 . A similar family with the same properties exists with linking number 0 .

I would like to thank W. B. R. Lickorish for several enlightening discussions and Robion Kirby for providing endless encouragement as well as suggesting the original problem. Thanks go also to both the referee and John Hughes for many helpful suggestions.

\section{Alexander polynomials of links.}

1. Definition of $\Delta_{L}(t)$. Given an oriented link $L$, we construct an infinite cyclic cover of $S^{3}-L$ in the following standard manner [R]. Let $F$ be a connected Seifert surface for $L$ compatible with the given orientation (say, so that an observer upright in the normal direction to $F$ and walking along $L$ sees $F$ to the left), and equipped with a bicollar $\stackrel{\circ}{F} \times[-1,1] \hookrightarrow S^{3}$. Let $\left\{Y_{i}=S^{3}-F \mid i \in \mathbf{Z}\right\}$ be infinitely many copies of $S^{3}-F$, and if $x \in \stackrel{F}{F}$, write $(x, t) \in \stackrel{\circ}{F} \times[-1,1]$. Then form

$$
M_{L}=\bigcup Y_{i} /\left(x_{i}, t\right) \sim\left(x_{i+1}, t-1\right)
$$

where $x_{i} \in Y_{i}$ and $x_{i+1} \in Y_{i+1}$ represent the same point in $\stackrel{\circ}{F}^{\circ}$ and where $t \in(0,1)$ (i.e., the positive side of $\dot{F}$ in $Y_{i}$ is glued to the negative side of $\stackrel{\circ}{F}$ in $Y_{i+1}$ ). 
Schematically,
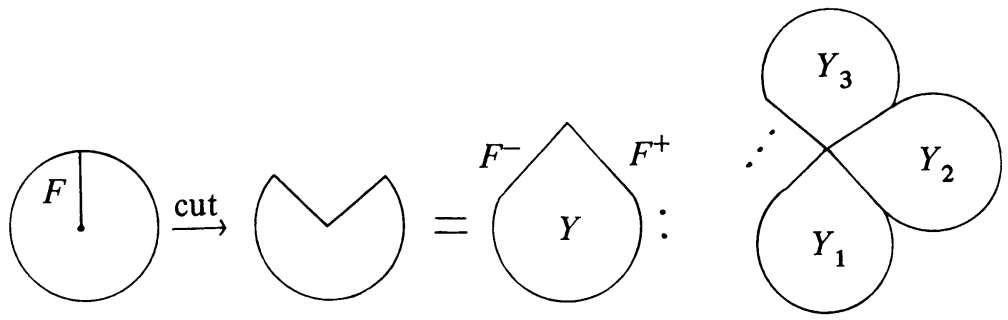

FIGURE 3

$M_{L}$ along with the obvious projection map is easily seen to be an infinite cyclic cover of $S^{3}-L$ (i.e., a covering space of $S^{3}-L$ with deck transformation group $\mathbf{Z}$ ). In general there are many infinite cyclic covers of the (unoriented) $S^{3}-L$, and we make the following

Proposition 1. The choice of infinite cyclic cover of $S^{3}-L$ depends only on the orientation of $L$ and not upon the choice of $F$.

Proof. This fact is well known and the proof will be omitted.

REMARK. In case $L$ is a knot there is only one infinite cyclic cover.

Recall now that $H_{1}\left(M_{L}\right)$ is a $\mathbf{Z}\left[t, t^{-1}\right]$-module, where $t$ acts by the induced action of the deck transformation $1 \in \mathbf{Z}$. As in $[\mathbf{R}]$, a Mayer-Vietoris argument shows that

$$
H_{1}\left(M_{L}\right)=\mathbf{Z}^{k} /\left(t V-V^{T}\right) \mathbf{Z}^{k}
$$

where $V$ is a Seifert matrix for $L$ arising from $F$. Furthermore, modulo multiplication by $\pm t^{n}, \operatorname{det}\left(t V-V^{T}\right)$ depends only on $M_{L}$ and not on the choice of $V$. Thus we have

Proposition 2. Let $L$ be an oriented link, and $V$ a Seifert matrix for $L$ arising from a compatibly oriented surface $F$. Then the Alexander polynomial

$$
\Delta_{L}(t)=\operatorname{det}\left(t V-V^{T}\right)
$$

is an isotopy invariant and modulo multiplication by $\pm t^{n}$ does not depend on the choice of $V$ or $F$.

REMARK. Although $\Delta_{L}(t)$ is an isotopy invariant of the oriented link $L$, it is not an invariant of the link complement. Since a given automorphism of $S^{3}-L$ may not preserve the preferred meridians corresponding to a choice of Seifert surface, the covers corresponding to these meridians need not be the same.

For example, twisting about the component labelled $A$ in Figure 4 describes a homeomorphism between the complements of the two links, each equipped with preferred orientations and preferred meridians $\mu_{1}, \mu_{2}, \mu_{3}$ and $\mu_{1}^{\prime}, \mu_{2}^{\prime}, \mu_{3}^{\prime}$. But $\mu_{1}$ is carried to $\mu=\mu_{1}+\mu_{2}+\mu_{3}$ by this homeomorphism, and so the infinite cyclic cover of the complement obtained by setting $\mu_{1}=\mu_{2}=\mu_{3}=1$ is not the same as that obtained by setting $\mu_{1}^{\prime}=\mu_{2}^{\prime}=\mu_{3}^{\prime}=1$. In fact, the polynomials of the above oriented links are $-(1-t)^{2}$ and $(1-t)^{4}+3 t(1-t)^{2}$ respectively. 


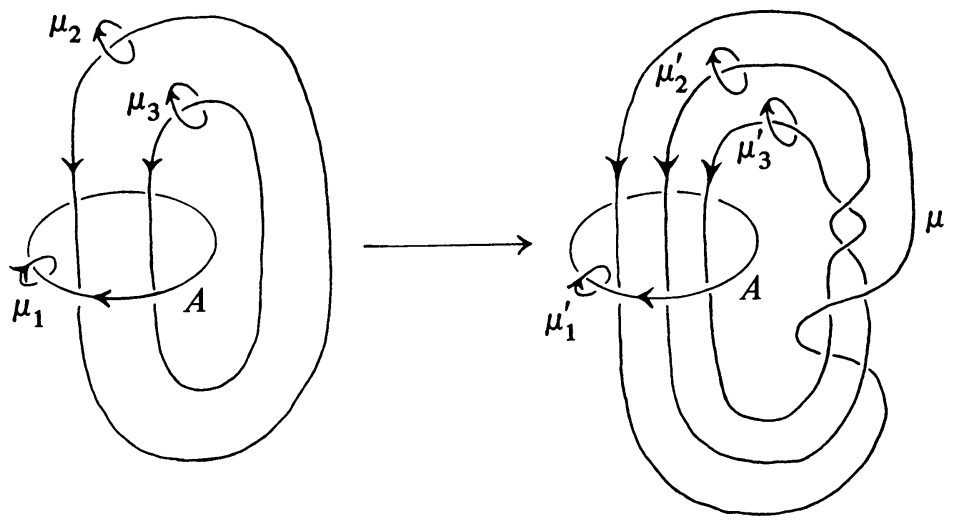

FIGURE 4

2. Conway's method for computing Alexander polynomials. Conway's idea is to calculate $\Delta_{L}(t)$ recursively using the following fact:

Fact. Any knot (or link) can be changed to the unknot (or unlink) by changing a finite number of overcrossings to undercrossings in some projection of the knot (or link).

To this end, we examine $\Delta_{L}(t)$ as a crossing is changed. To be definite, let $L_{l}$ be a link in which a left-handed crossing occurs, let $L_{r}$ be the same link with the crossing changed to a right-handed crossing, and let $L_{s}$ be $L_{l}$ with the crossing "smoothed" (see Figure 5). Each shaded surface is part of the Seifert surface given by a standard algorithm [R], and each $\Delta_{L}$ is to be calculated from these chosen surfaces.

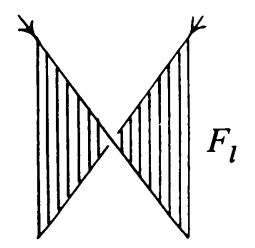

$L_{l}$

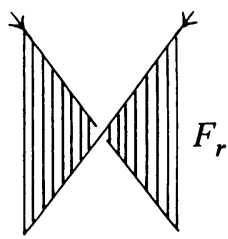

$L_{r}$

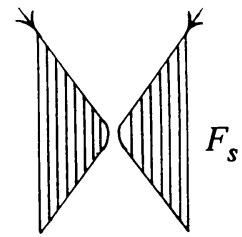

$L_{S}$

Figure 5
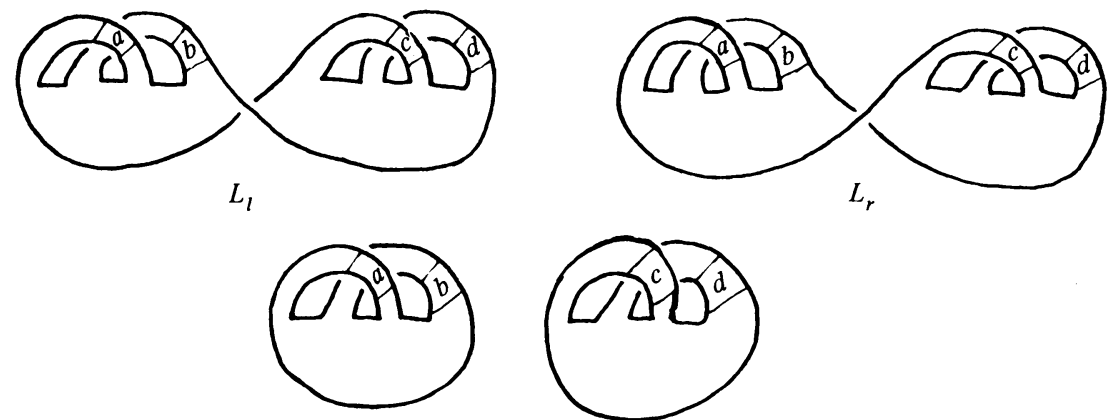

$L_{s}$

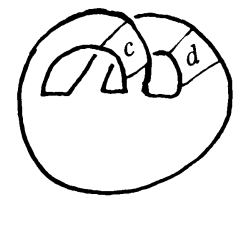

Figure 6 
LeMma 3. $\Delta_{L_{l}}(t)=\Delta_{L_{r}}(t)+(t-1) \Delta_{L_{s}}(t)$.

Proof. If there is no basis element of $H_{1}\left(F_{l}\right)$ through the given crossing, then $L_{l}$ must split as a direct sum with $L_{l}=L_{r}$ and $L_{s}$ disconnected (so that $\Delta_{L_{l}}(t)=\Delta_{L_{r}}(t)$ ) (see Figure 6). A connected surface $F$ for $L_{s}$ is given by tubing together the previous two disjoint surfaces (see Figure 7).

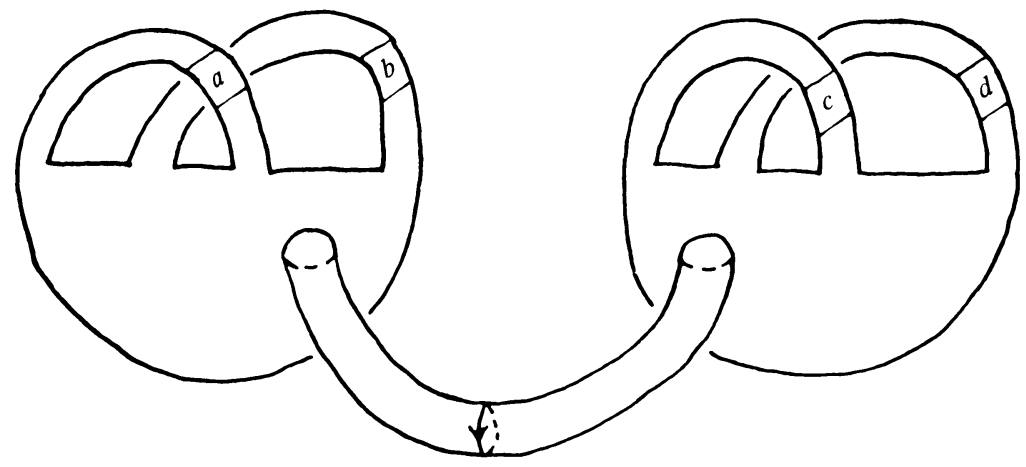

FIGURE 7

Using the meridian $\alpha$ of the tube as a basis element for $H_{1}(F)$, we see that the row and column corresponding to $\alpha$ in the Seifert matrix $V$ is all 0 's, and so $\Delta_{L_{s}}(t)=$ $\operatorname{det}\left(t V-V^{T}\right)=0$. Then since $\Delta_{L_{l}}(t)=\Delta_{L_{r}}(t)$, the lemma is true in this case.

If there is a basis of $H_{1}\left(F_{l}\right)$ containing an element through the indicated crossing, then we can rechoose the basis so that only one element $\alpha$ passes through the crossing. Then the Seifert matrices $V_{l}, V_{r}$, and $V_{s}$ of $L_{l}, L_{r}$, and $L_{s}$ respectively satisfy

$$
V_{r}=\alpha\left(\begin{array}{c|c}
\alpha \\
a & * \\
\hline * & V_{s}
\end{array}\right), \quad V_{l}=\left(\begin{array}{c|c}
a+1 & * \\
\hline * & V_{s}
\end{array}\right)
$$

so that

$$
\Delta_{L_{l}}(t)=\operatorname{det}\left(t V_{l}-V_{l}^{T}\right)=\operatorname{det}\left(\begin{array}{c|c}
(t-1)(a+1) & * \\
\hline * & t V_{s}-V_{s}^{T}
\end{array}\right),
$$

and expanding about the first row,

$$
=\operatorname{det}\left(\begin{array}{c|c}
(t-1) a & * \\
\hline * & t V_{s}-V_{s}^{T}
\end{array}\right)+(t-1) \operatorname{det}\left(t V_{s}-V_{s}^{T}\right) .
$$

At first glance, this seems to define $\Delta_{L}(t)$ recursively; simply change $L$ to the unlink by changing crossings and smoothing (an easy induction on the number of crossings ensures that this can be done), and compute $\Delta_{L}(t)$ at each stage by Lemma 3. For concreteness, start with $\Delta($ unknot $)=1$. However, this scheme does not quite work; the Alexander polynomial is defined only up to multiplication by $\pm t^{n}$, and so is only a representative of the set $S=\left\{ \pm t^{s} \operatorname{det}\left(t V-V^{T}\right) \mid s \in \mathbf{Q}\right\}$. So, the recursion gives a well-defined choice of $\Delta_{L}(t)$ only if we can choose for all $L$ a specific 
canonical representative for $\Delta_{L}(t)$, and only if this choice is preserved by the recursion formula. A natural choice is to assume $\Delta_{L}(t)$ is symmetrized, i.e., of the form

$$
a_{\alpha} t^{\alpha}+a_{\alpha-1} t^{\alpha-1}+\cdots+a_{-\alpha+1} t^{-\alpha+1}+a_{-\alpha} t^{-\alpha}, \quad \text { with } a_{\alpha}, a_{-\alpha} \neq 0 .
$$

However, (1) does not preserve this choice. To fix matters up, we redefine $\Delta_{L}(t)$ as follows. Let $F$ be the surface from which $V$ arises and WLOG suppose $F$ is built from a disk by adding $b$ bands (it is not hard to show that $b=2 g+k-1$, where $g=$ genus $F$ and $k$ is the number of components of $L$ ). Then $V$ is a $b \times b$ matrix, and we redefine

$$
\begin{aligned}
\Delta_{L}(t) & =t^{-b / 2} \operatorname{det}\left(t V-V^{T}\right) \\
& =\operatorname{det}\left(t^{-1 / 2}\left(t V-V^{T}\right)\right)=\operatorname{det}\left(t^{1 / 2} V-t^{-1 / 2} V^{T}\right) .
\end{aligned}
$$

With this definition, $\Delta_{L}(t)$ is a representative of $S$ and is clearly symmetric as in (2), although $\alpha$ need not be an integer. Furthermore, repeating the calculation leading to (1) yields

$$
\Delta_{L_{l}}(t)=\Delta_{L_{r}}(t)+\left(t^{1 / 2}-t^{-1 / 2}\right) \Delta_{L_{s}}
$$

so that our recursion now preserves symmetry (i.e., if any two of $\Delta_{L_{l}}, \Delta_{L_{r}}$, and $\Delta_{L_{\mathrm{s}}}$ are symmetric, then so is the third). Thus the recursion gives us one of the two symmetrized representatives $\pm \Delta_{L}(t)$. In fact, we get a unique (even up to \pm ) representative of $S$, as shown by the following three lemmas and corollary.

Lemma 4. Let $K$ be a knot. Then $\Delta_{K}(1)=+1$ for any choice of Seifert surface for $K$.

Proof. $\Delta_{K}(t)=\operatorname{det}\left(t^{1 / 2} V-t^{-1 / 2} V^{T}\right)$, so $\Delta_{K}(1)=\operatorname{det}\left(V-V^{T}\right)$. But $V-V^{T}$ is the intersection form of $F$ and is a direct sum of copies of $\left(\begin{array}{cc}0 & 1 \\ -1 & 0\end{array}\right)$ for some basis of $H_{1}(F)$ (see [R, p. 202]).

LEMma 5. $\Delta_{K}(t)$ as defined above gives a unique representative in $S$.

Proof. The condition $\Delta_{K}(1)=1$ determines which of $\Delta_{K}(t)$ is chosen.

Lemma 6. Let $F_{1}$ and $F_{2}$ be two Seifert surfaces for a link $L$ of two components, and let $\Delta_{i}(t)$ be the symmetrized form of $\operatorname{det}\left(t^{1 / 2} V_{i}-t^{-1 / 2} V_{i}^{T}\right)$, where $V_{i}$ is the Seifert matrix arising from $F_{i}$. Then $\Delta_{1}(t)=\Delta_{2}(t)$.
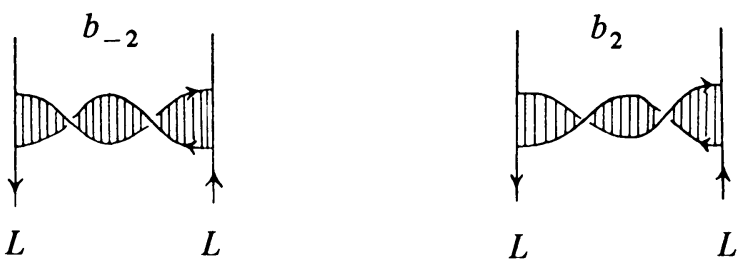

FIGURE 8 
Proof. Let $b_{n}$ be a band containing $n$ half-twists added to $L$ so that $L \cup \partial b_{2 n}$ is a knot and so that $F_{1} \cup b_{2 n}$ is a Seifert surface for $L \cup \partial b_{2 n}$ (see Figure 8). Then (3) gives

$$
\begin{aligned}
\Delta\left(L \cup \partial b_{2}\right) & =\Delta\left(L \cup \partial b_{0}\right)+\left(t^{1 / 2}-t^{-1 / 2}\right) \Delta_{1}(L) \\
& =\left(\Delta\left(L \cup \partial b_{-2}\right)+\left(t^{1 / 2}-t^{-1 / 2}\right) \Delta_{1}(L)\right)+\left(t^{1 / 2}-t^{-1 / 2}\right) \Delta_{1}(L) .
\end{aligned}
$$

So

$$
\Delta_{1}(L)=\frac{\Delta\left(L \cup \partial b_{2}\right)-\Delta\left(L \cup \partial b_{-2}\right)}{2\left(t^{1 / 2}-t^{-1 / 2}\right)} .
$$

If $b_{n}$ does not intersect $F_{2}$, we can form a new surface $F_{2} \cup b_{2 n}$ for $L \cup \partial b_{2 n}$, calculate as above, and find

$$
\Delta_{2}(L)=\frac{\Delta\left(L \cup \partial b_{2}\right)-\Delta\left(L \cup \partial b_{-2}\right)}{2\left(t^{1 / 2}-t^{-1 / 2}\right)} .
$$

Since, by Lemma 5, $\Delta$ of a knot is independent of the surface chosen, $\Delta_{1}(L)=\Delta_{2}(L)$ in this case as claimed.

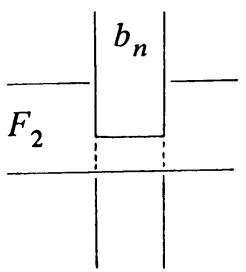

FigURE 9
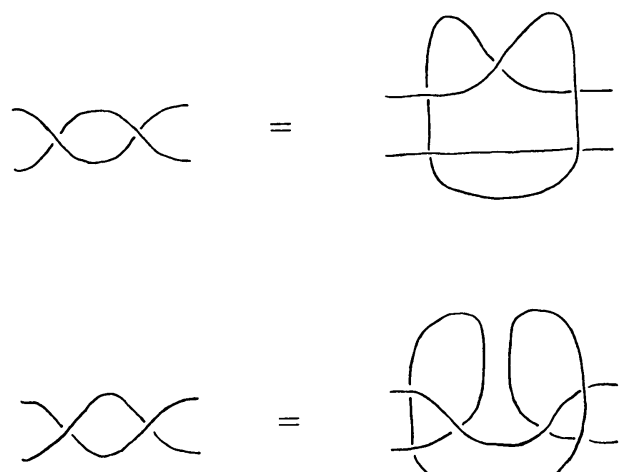

$$
=
$$

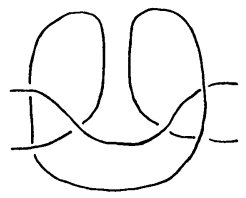

Figure 10 

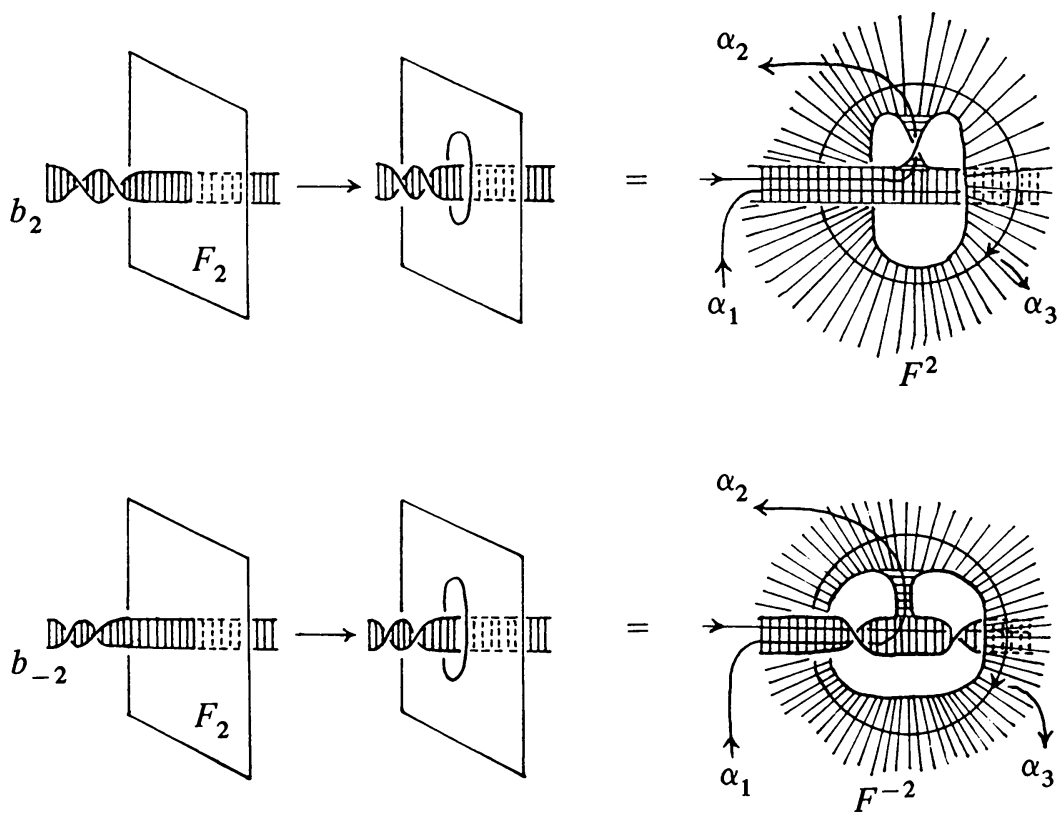

FIGURE 11

Suppose however that $b_{n}$ intersects $F_{2}$ in (for simplicity's sake) one ribbon intersection (see Figure 9). First note the isotopies in Figure 10. Then form new Seifert surfaces $F^{2}$ and $F^{-2}$ for $L \cup \partial b_{2}$ and $L \cup \partial b_{-2}$ respectively by first puncturing $F_{2}$ and filling in with the surfaces in Figure 11. Note that if necessary we can replace Figure 12 by Figure 13 so that the surfaces $F^{2}$ and $F^{-2}$ are indeed orientable.

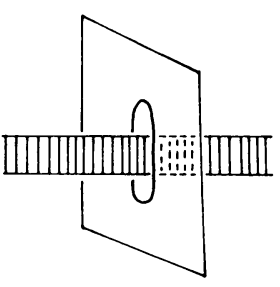

FIGURE 12

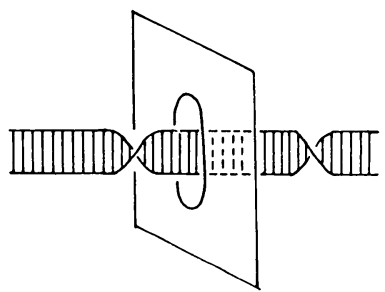

FIGURE 13

Then using the elements $\alpha_{1}, \alpha_{2}, \alpha_{3}$ of $H_{1}\left(F^{i}\right)$ indicated, the corresponding Seifert matrices are

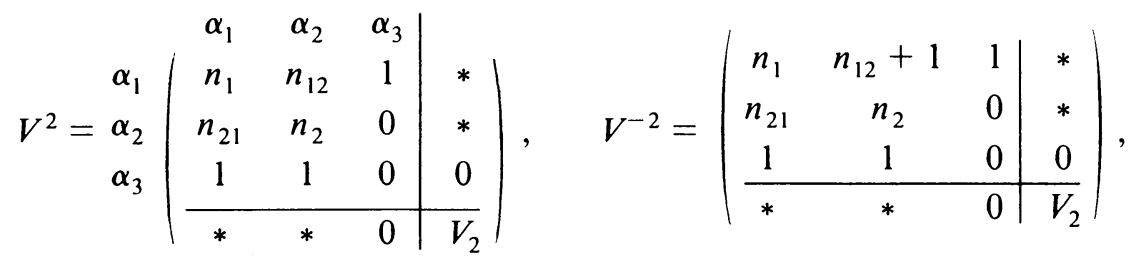

where the $n_{i}$ 's are integers and $V_{2}$ is the Seifert matrix arising from $F_{2}$. 
Then expanding about the rows indicated by arrows,

$$
\begin{aligned}
& \operatorname{det}\left(t^{1 / 2} V^{-2}-t^{-1 / 2} V^{-2^{T}}\right) \\
& =\operatorname{det}\left(\begin{array}{ccc|c}
n_{1}\left(t^{1 / 2}-t^{-1 / 2}\right) & n_{12} t^{1 / 2}-n_{21} t^{-1 / 2}+t^{1 / 2} & t^{1 / 2}-t^{-1 / 2} & * \\
n_{21} t^{1 / 2}-n_{12} t^{-1 / 2}-t^{1 / 2} & n_{2}\left(t^{1 / 2}-t^{-1 / 2}\right) & -t^{-1 / 2} & * \\
t^{1 / 2}-t^{-1 / 2} & t^{1 / 2} & 0 & 0 \\
\hline * & * & 0 & t^{1 / 2} V_{2}-t^{-1 / 2} V_{2}^{T}
\end{array}\right) \\
& =\operatorname{det}\left(\begin{array}{ccc|c}
n_{1}\left(t^{1 / 2}-t^{-1 / 2}\right) & n_{12} t^{1 / 2}-n_{21} t^{-1 / 2} & t^{1 / 2}-t^{-1 / 2} & * \\
n_{21} t^{1 / 2}-n_{12} t^{-1 / 2}-t^{-1 / 2} & n_{2}\left(t^{1 / 2}-t^{-1 / 2}\right) & t^{-1 / 2} & * \\
t^{1 / 2}-t^{-1 / 2} & t^{1 / 2} & 0 & 0 \\
\hline * & * & 0 & t^{1 / 2} V_{2}-t^{-1 / 2} V_{2}^{T}
\end{array}\right) \leftarrow \\
& -t^{1 / 2} \operatorname{det}\left(\begin{array}{cc|c}
n_{21} t^{1 / 2}-n_{12} t^{-1 / 2}-t^{-1 / 2} & -t^{-1 / 2} & * \\
t^{1 / 2}-t^{-1 / 2} & 0 & 0 \\
\hline * & 0 & t^{1 / 2} V_{2}-t^{-1 / 2} V_{2}^{T}
\end{array}\right) \leftarrow \\
& =\operatorname{det}\left(t^{1 / 2} V^{2}-t^{-1 / 2} V^{2^{T}}\right)+t^{-1 / 2} \operatorname{det}\left(\begin{array}{cc|c}
n_{12} t^{1 / 2}-n_{21} t^{-1 / 2} & t^{1 / 2}-t^{-1 / 2} & * \\
t^{1 / 2} & 0 & 0 \\
\hline * & 0 & t^{1 / 2} V_{2}-t^{-1 / 2} V_{2}^{T}
\end{array}\right) \\
& -\left(t^{1 / 2}-t^{-1 / 2}\right) \operatorname{det}\left(t^{1 / 2} V_{2}-t^{-1 / 2} V_{2}^{T}\right) \\
& =\operatorname{det}\left(t^{1 / 2} V^{2}-t^{-1 / 2} V^{2^{T}}\right)-2\left(t^{1 / 2}-t^{-1 / 2}\right) \operatorname{det}\left(t^{1 / 2} V_{2}-t^{-1 / 2} V_{2}^{T}\right) \text {. }
\end{aligned}
$$

Thus, using the surfaces $F^{2}, F^{-2}$, and $F_{2}$ we have again formula (4). Then as for the case where $b_{n}$ does not intersect $F_{2}$, we see that $\Delta_{2}(L)$ and $\Delta_{1}(L)$ can be written as identical expressions in the polynomials of the knots $L \cup \partial b_{2}$ and $L \cup \partial b_{-2}$; since such polynomials are independent of which surface is chosen for the computation, $\Delta_{2}(L)=\Delta_{1}(L)$ as claimed.

COROllary 7. The symmetrized $\Delta_{L}(t)=\operatorname{det}\left(t^{1 / 2} V-t^{-1 / 2} V^{T}\right)$ is independent (even up to \pm ) of $V$.

Proof. Induct on the number of components of $L$, banding together two different components and using the above argument at each stage of the induction.

For convenience, we set $z=t^{1 / 2}-t^{-1 / 2}$. Note that by (3) any $\Delta_{L}(t)$ may be written as a unique polynomial $\nabla_{L}(z)$, as long as we set $\nabla($ unknot $)=1$. So finally, we have a recursive scheme

$$
\nabla_{L_{l}}(z)=\nabla_{L_{r}}(z)+z \nabla_{L_{s}}(z), \quad \nabla_{\text {unknot }}(z)=1
$$

$\nabla_{L}(z)$ is called the Conway potential function of $L$.

REMARK. Kauffman [K] has proven the independence of $\nabla_{L}(z)$ from the choice of Seifert surface by noting that it is an invariant of $S$-equivalence, a notion not used in Lemma 6.

3. Examples. We will use the following conventions. An asterisk (*) by a crossing indicates that the crossing is to be changed and smoothed in the next step as in the previous sections. We will indicate the status of three links as being $L_{l}, L_{r}$, and $L_{s}$ by

$$
L_{l}=L_{r} \oplus L_{s} \quad \text { or } \quad L_{r}=L_{l} \ominus L_{s}
$$


Note that $\oplus$ and $\Theta$ are not commutative or associative. We will write $\nabla_{L}(z)$ as $\nabla(L)$ or $\nabla$ (a picture of $L)$ according to convenience, and we will brutally abuse notation by writing a picture of $L$ to mean either $L$ or $\nabla_{L}(z)$ depending on the context.

(a)

$$
1=\nabla(\bullet)=\nabla(\infty)=\nabla(\infty)+z \nabla(\infty)
$$

by $(5)$, and so $\nabla(\diamond \diamond)=0$.

Similarly, for any links $L_{1}$ and $L_{2}$ separated in $S^{3}$ by an $S^{2}, \nabla\left(L_{1} \cup L_{2}\right)=0$. This was proved geometrically in Lemma 3.

(b)

$$
\nabla(\text { 䒜 })=\nabla(00)+z \nabla(0)=z \text {, }
$$

by (5) and $\S 3 \mathrm{a}$; we write $\circlearrowright=(00) \oplus \Theta$.

(c)

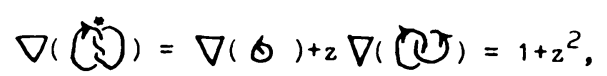

and we write $\left(\hat{D}=\Theta \oplus\right.$. Note that $1+z^{2}=1+\left(t^{1 / 2}-t^{-1 / 2}\right)^{2}=t-1-$ $t^{-1}$ as expected.

$$
\begin{aligned}
& \nabla(\text { ठ) }=\nabla(\text { の })-2 \nabla(\text { の })=-z \text {. }
\end{aligned}
$$

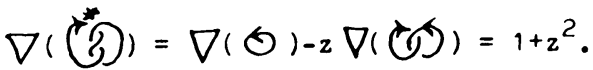

Note that interchanging $L_{l}$ and $L_{r}$ in (5) has the effect of changing the + to a -

(e) Define

$$
\nabla_{n}(z)=\nabla(\Re) \text { 年. }
$$

Then $\nabla_{n}=\nabla_{n+2}+z \nabla_{n+1}$.

REMARKS. (1) When calculating $\nabla_{L}(z)$ in this fashion it is not necessary to draw a Seifert surface for $L$ or to write down a Seifert matrix. This represents an enormous savings of time and effort in practical applications.

(2) By construction, when $\nabla_{L}(z)$ is of degree $d$ in $z$ and is converted to $\nabla_{L}(t)$ by the substitution $z=t^{1 / 2}-t^{-1 / 2}$, the result is a symmetrized polynomial in $t$ of degree $\frac{1}{2} d: a_{d / 2} t^{d / 2}+a_{d / 2-1} t^{d / 2-1}+\cdots+a_{-d / 2+1} t^{-d / 2+1}+a_{-d / 2} t^{-d / 2}$. Multiplying by $t^{d / 2}$, we see that the degree of $\Delta_{L}(t)$ as a polynomial in $\mathbf{Z}[t]$ with constant coefficient $a_{-d / 2}$ is the degree $d$ of $\nabla_{L}(z)$ in $z$. But an Euler characteristic argument shows that the number of bands of $F$ (and hence the size of the matrix $V$ ) is $2 g+k-1$, where $k$ is the number of components of $L$ and $g$ is the genus of $F$. Thus if $g_{L}$ is the genus of the link $L$, then $2 g_{L}+k-1 \geqslant \operatorname{deg} \nabla_{L}(z)$. In the case of a $\operatorname{knot} K$,

$$
2 g_{K} \geqslant \operatorname{deg} \nabla_{K}(z)=\operatorname{deg} \Delta_{K}(t) .
$$

This inequality will be used later to distinguish the two lifts.

4. Computational lemmas.

LEMMA 8. Let $K$ be a knot and $L$ be a link. Then

(a) $\Delta_{K}(1)=\nabla_{K}(0)=1$.

(b) If L has more than one component, then $\nabla_{L}(0)=0$.

(c) $\nabla_{K}(z)$ is a polynomial in $z^{2}$. 
(d) $\Delta_{K}(t)$ and $\nabla_{K}(z)$ are independent of the orientation of $K$; in fact, $\nabla_{L}(z)$ does not change when the orientations are simultaneously reversed on all components of $L$ (recall that changing the orientation of a strand corresponds to a change in choice of infinite cyclic cover ).

(e) Substituting $t^{1 / 2}-t^{-1 / 2}$ for $z$ in $\nabla_{K}(z)$ gives the symmetrized $\Delta_{K}(t)$; furthermore, in the notation of (2), $a_{\alpha}=a_{-\alpha}$ (i.e., $\left.\Delta_{K}(t)=\Delta_{K}\left(t^{-1}\right)\right)$.

REMARK. (a) and (b) are equivalent to the fact that if $F$ is a Seifert surface for $L$, then the determinant of the intersection matrix of $F$ is

$$
\begin{cases}1, & L \text { a knot, } \\ 0, & L \text { has more than one component. }\end{cases}
$$

Proof. (a) When $t=1$, we have $z=0$; therefore this statement is Lemma 4 .

(b) $L$ can be changed to the unlink of greater than one component by changing crossings. Setting $z=0$ and using (5) shows $\nabla_{L}(0)=0$.

(c) If $L$ has more than one component, (b) shows that $\nabla_{L}(z)$ is a multiple of $z$. An induction on the crossing number using this fact and (5) finishes the proof.

(d) Changing the orientation on all strands of a link does not change the sign of each crossing, and so the recursion defining $\nabla_{L}(z)$ remains the same.

(e) By (c), $\nabla_{K}(z)=\nabla_{K}(-z)$, i.e., $\Delta_{K}(t)=\Delta_{K}\left(t^{-1}\right)$.

LEMMA 9. Recall that

$$
\nabla_{\mathrm{n}}=(\vartheta \ln ), \quad \mathrm{n} \in \mathbb{Z} .
$$

Then

(a) $\nabla_{2 n}=-\nabla_{-2 n}$,

(b) $\nabla_{2 n+1}=\nabla_{-(2 n+1)}$,

(c) $\operatorname{deg} \nabla_{n}(z)=|n|-1$.

Proof. (a) and (b). Easy induction, using (5). The examples in $\S 3$ show the lemma is true for $k=1$. Suppose it is true for $k \leqslant n$; then

$$
\begin{aligned}
\nabla_{2(n+1)} & =\nabla_{2 n+2}=\nabla_{2 n}-z \nabla_{2 n+1}=-\nabla_{-2 n}-z \nabla_{-(2 n+1)} \\
& =-\left(\nabla_{-2 n}+z \nabla_{-2 n-1}\right)=-\nabla_{-2(n+1)} .
\end{aligned}
$$

Similarly, $\nabla_{2(n+1)+1}=\nabla_{-(2(n+1)+1)}$. Thus (a) and (b) are proven for $n \geqslant 0$; a similar argument holds for $n \leqslant 0$.

(c) Straightforward induction using Example 3(e).

The following lemma gives formulas for $\nabla$ of a link in which $n$ occurs. Although useful in specific examples, it will not be used later and so will not be proven here (the proof, of course, is by induction and (5)).

LEMMA 10. (a) Let 简 occur in a link L. Let $L_{j}$ be the link obtained from $L$ by replacing $n$ by $j$. Then

$$
\nabla_{L}=\nabla_{n} \nabla_{L, 1}+\nabla_{n+1} \nabla_{L_{0}},
$$

with $a+$ or - as $n$ is positive or negative. 
(b) Similarly, suppose smoothing the crossing. Then

$$
\nabla_{L}=\nabla_{L_{0}}+[n / 2] z \nabla_{L_{s}}
$$

COROLlaRY 11. Let L be a link, and let $\#$ denote connected sum. Then

$$
\nabla(\mathrm{L} \# \hat{\mathrm{n}} \mathrm{O})=\nabla_{\mathrm{n}} \nabla_{\mathrm{L}} \text {. }
$$

Proof. By the lemma,

$$
\nabla(\mathrm{L} \# \mathrm{O} \mathrm{\theta})=\nabla_{\mathrm{n}} \nabla_{\mathrm{L}}+\nabla_{\mathrm{n} \neq 1} \nabla(\mathrm{L} \cup \circlearrowright) \text {. }
$$

But the second term is zero by example 3(a).

REMARK. This is a special case of $\nabla\left(L_{1} \sharp L_{2}\right)=\nabla_{L_{1}} \nabla_{L_{2}}$ which is well known for knots. It will follow later from Corollary 16.

5. Conway's room theory. Following and generalizing Conway [C2], we develop a powerful method to calculate $\nabla_{L}$.

Definition. A room $R$ is a connected domain (usually a rectangle) in $\mathbf{R}^{2}$ equipped with an equal number of oriented ingoing and outgoing strands:

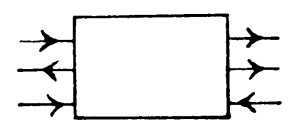

The skein of a room, $S(R)$, is the set of all collections of strands in the room which connect ingoing to outgoing strands. For example, Figure 14 is an element of the skein of the above room.

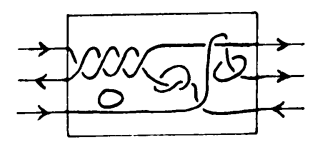

FIGURE 14

In this discussion we will always take $R$ to be the following room with $n$ ingoing and outgoing strands:

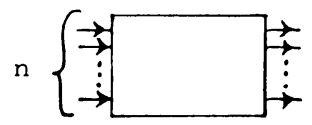

Our arguments generalize in obvious ways to other rooms.

Definition. Let $S_{n}$ denote the full symmetric group on $n$ letters. Given a skein element $s$, we assign a permutation $\pi(s) \in S_{n}$ by numbering the strands of $R$ :

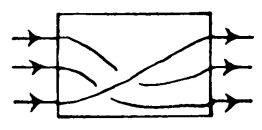

Now pick an ordering $\left\{\pi_{i}\right\}_{i=1, n \text { ! }}$ of $S_{n}$ and fix a choice of skein element $s_{\pi_{i}}$ for each $\pi_{i} \in S_{n}$ such that $\pi\left(s_{\pi_{i}}\right)=\pi_{i}$, and such that $s_{\pi_{i}}$ contains no free component. 
EXAMPLE. $n=2$.
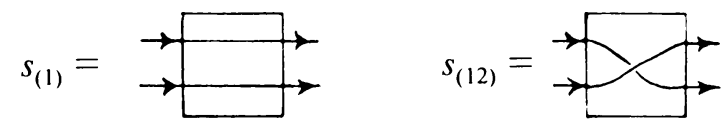

$n=3$.

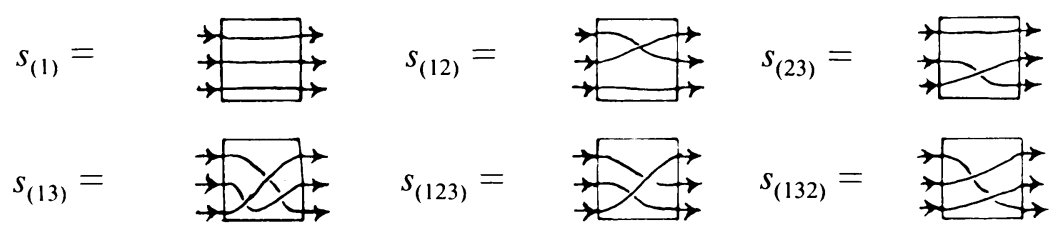

Definition. Define a binary operation on $S=S(R)$ by juxtaposition:

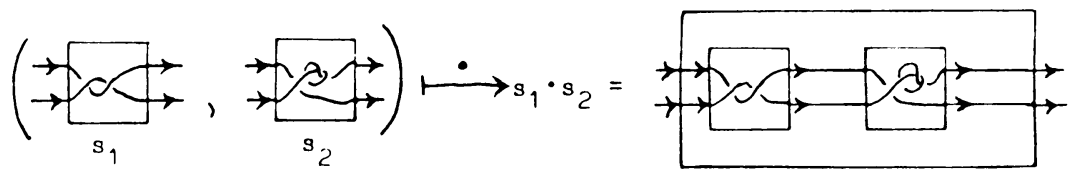

Note that $\pi\left(s_{1} \cdot s_{2}\right)=\pi\left(s_{1}\right) \pi\left(s_{2}\right) \in S_{n}$.

Now let $s_{l}$ be a skein element in which a left-handed crossing appears, and let $s_{r}$ (respectively $s_{s}$ ) be the same element with the crossing changed to a right (respectively smoothed) crossing. We call $\left(s_{l}, s_{r}, s_{s}\right)$ a skein triple.

Definition. Let $F$ be the quotient field of $\mathbf{Z}[z]$, let $V(S)$ be the vector space generated by $S$ over $F$, and let $N(S)$ be the vector space generated by $\left\{s_{l}-s_{r}-z s_{s} \mid\right.$ $\left(s_{l}, s_{r}, s_{s}\right)$ is a skein triple $\}$ over $F$. Then define the linearization $L(S)$ by $L(S)=$ $V(S) / N(S)$ (i.e., we impose the previous recursion relations on $V(S)$ ).

$L(S)$ inherits a vector space structure from $V(S)$, and is in fact an algebra under the extension of - to $L(S)$, since $\cdot$ preserves the relations in $N(S)$ :

$$
s_{1} \cdot\left(s_{r}+z s_{s}\right)=s_{1} \cdot s_{r}+z s_{1} \cdot s_{s}=s_{1} \cdot s_{l} \text {. }
$$

Definition. Define $L_{0}(S), N_{0}(S)$, and $V_{0}(S)$ the same as for $L(S), N(S)$, and $V(S)$ respectively, using $\mathbf{Z}[z]$ instead of $F$.

LEMMA 12. Setting $z \mapsto 0$ and $s \mapsto \pi(s)$ defines an algebra homomorphism

$$
L_{0}(S) \stackrel{e}{\rightarrow} \mathbf{Z}\left[S_{n}\right],
$$

where $\mathbf{Z}\left[S_{n}\right]$ is the group ring of $S_{n}$.

Proof. An element $\alpha$ of $V_{0}(S)$ is a linear combination of elements of $S$ with coefficients in $\mathbf{Z}[z]$. The above map takes $\alpha$ into $\mathbf{Z}\left[S_{n}\right]$, and we must show $e(N)=0$. But $e\left(s_{l}-s_{r}-z s_{s}\right)=e\left(s_{l}\right)-e\left(s_{r}\right)=\pi\left(s_{l}\right)-\pi\left(s_{r}\right)=0$.

Furthermore, for skein elements $s_{1}$ and $s_{2}, e\left(s_{1} \cdot s_{2}\right)=\pi\left(s_{1} \cdot s_{2}\right)=\pi\left(s_{1}\right) \pi\left(s_{2}\right)=$ $e\left(s_{1}\right) e\left(s_{2}\right)$ so that $e$ may be extended to an algebra homomorphism.

Proposition 13. $\left\{s_{\pi} \mid \pi \in S_{n}\right\}$ defined previously is a basis for $L(S)$. 
Proof. By a skein move we mean either the equating of $s_{l}$ with $s_{r}+z s_{s}$ (or of $s_{r}$ with $s_{l}-z s_{s}$ ) as is done on dividing $V(S)$ by $N(S)$, or the geometric analogue of changing and smoothing a crossing. A moment's thought shows that any skein element may be decomposed by skein moves (i.e., rewritten in $L(S)$ ) to a linear combination over $\mathbf{Z}[z]$ of elements of $\left\{s_{\pi} \mid \pi \in S_{n}\right\}$ or skein elements containing a free component separated by an $S^{2}$.

EXAMPLE.

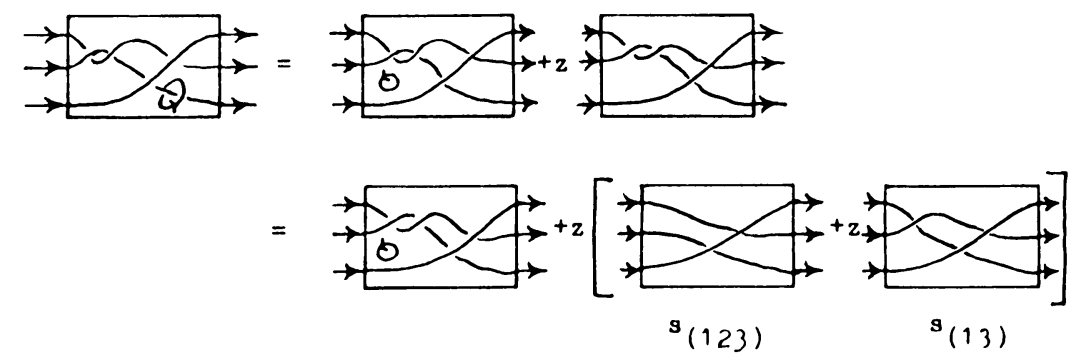

But as in §3a, skein elements with separated free components are zero in $L(S)$. Thus, $\left\{s_{\pi_{i}} \mid \pi_{i} \in S_{n}\right\}$ at least spans $L(S)$.

Suppose, however, that $\sum a_{i}(z) s_{\pi_{i}}=0$, for $a_{i}(z) \in F$. Then by multiplying by polynomials if necessary, we may assume $a_{i}(z) \in \mathbf{Z}[z]$ and that some $a_{i}(0) \neq 0$. So $\sum a_{i}(z) s_{\pi_{i}} \in L_{0}(S)$, and $e\left(\sum a_{i}(z) s_{\pi_{i}}\right)=\Sigma a_{i}(0) \pi_{i}=0$, a contradiction as $\left\{\pi_{i} \mid \pi_{i} \in S_{n}\right\}$ is a basis for $\mathbf{Z}\left[S_{n}\right]$.

In particular, we now have

COROLlary 14. Any skein element s can be expressed uniquely as a linear combination of the $s_{\pi}$, so that $\operatorname{dim}_{F} L(S)=n$ !.

Definition. Given skein elements $s$ and $x$, define the dual $s^{*}$ by

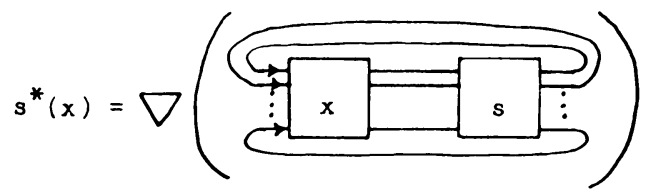

$s^{*}$ may be extended linearly to all of $L(S)$; note that the so-defined $s^{*}$ preserves skein moves:

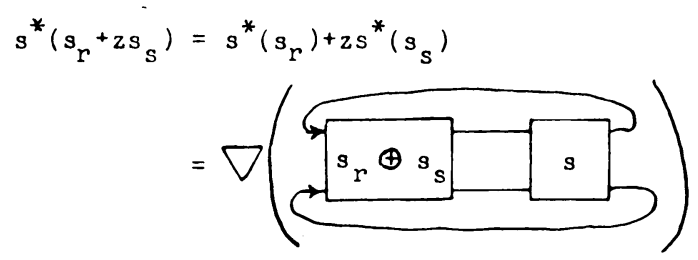

So we get a dual element $s^{*}: L(S) \rightarrow \mathbf{Z}[z] \subseteq F$.

Let $M_{n}$ be the $n ! \times n !$ matrix defined by $\left(M_{n}\right)_{i j}=s_{i}^{*}\left(s_{j}\right)$. Then $\operatorname{det}\left(M_{n}\right) \neq 0$ iff $\left\{s_{i}^{*}\right\}$ is a basis of $L(S)^{*}$. Direct computation shows that $\operatorname{det}\left(M_{n}\right) \neq 0$ for $n=2$ and 
3 ; using the above basis, $M_{n}$ is respectively

$$
\begin{aligned}
M_{2} & =\left(\begin{array}{ll}
0 & 1 \\
1 & z
\end{array}\right), \\
M_{3} & =\left(\begin{array}{lccccc}
0 & 0 & 0 & z & 1 & 1 \\
0 & 0 & 1 & 1+z^{2} & z & z \\
0 & 1 & 0 & 1+z^{2} & z & z \\
z & 1+z^{2} & 1+z^{2} & 3 z^{2}+z^{4} & 2 z+z^{3} & 2 z+z^{3} \\
1 & z & z & 2 z+z^{3} & 1+z^{2} & z^{2} \\
1 & z & z & 2 z+z^{3} & z^{2} & 1+z^{2}
\end{array}\right), \\
M_{3}^{-1} & \left.=\frac{-1}{z^{2}+4} \mid \begin{array}{cccccc}
-z^{4}-3 z^{2}+2 & z^{3}+3 z & z^{3}+3 z & z & -z^{2}-2 & -z^{2}-2 \\
z^{3}+3 z & 2 & -z^{2}-2 & -2 & z & z \\
z^{3}+3 z & -z^{2}-2 & 2 & -2 & z & z \\
z & -2 & -2 & 2 & -z & -z \\
-z^{2}-2 & z & z & -z & -2 & z^{2}+2 \\
-z^{2}-2 & z & z & -z & z^{2}+2 & -2
\end{array}\right),
\end{aligned}
$$

$\operatorname{det}\left(M_{3}\right)=-\left(z^{2}+4\right)$.

Thus $\left\{s_{i}^{*}\right\}$ is a dual base for $L(S)$ when $n=2$ or 3 .

Conjecture. $\operatorname{det}\left(M_{n}\right) \neq 0$, all $n$.

Remarks. Since $s_{i}^{*}\left(s_{j}\right) \in \mathbf{Z}[z]$, one may similarly define dual elements to $e\left(L_{0}(S)\right)=\mathbf{Z}\left[S_{n}\right]$, by setting $\bar{s}_{i}^{*}\left(s_{j}\right)=e\left(s_{i}^{*}\left(s_{j}\right)\right)$ (recall that $e$ has the effect of setting $z=0$ ). Then the linear independence of the $\bar{s}_{1}^{*}$ is equivalent to the nonvanishing of the determinant of the matrix $\bar{M}_{n}$ obtained by setting $z=0$ in the entries of $M_{n}$; this in turn implies that $\operatorname{det}\left(M_{n}\right) \neq 0$. However, recalling the fact that

$$
\nabla_{L}(0)= \begin{cases}0, & L \text { has more than one component } \\ 1, & L \text { a knot }\end{cases}
$$

and noting that

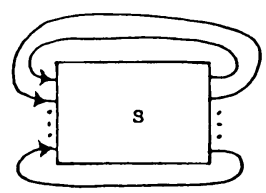

is a knot iff $\pi(s)$ is an $n$-cycle, we see that

$$
M_{i j}= \begin{cases}1, & \pi_{i} \pi_{j} \text { an } n \text {-cycle } \\ 0, & \text { otherwise }\end{cases}
$$

Then $M$ is similar to $c=\sum$ (n-cycles) under the regular representation in $\mathbf{C}\left[S_{n}\right]$. Using elementary representation theory as in [I] one may in fact show that $\operatorname{det}\left(M_{n}\right)=0$ for $n \geqslant 4$. This means that the $s_{i}^{*}$ do not form a basis for $\mathbf{Z}\left[S_{n}\right]^{*}$, although we conjecture that $\left\{s_{i}^{*}\right\}$ is a basis for $L(S)^{*}$.

Accordingly, the following discussion holds when $\operatorname{det}\left(M_{n}\right) \neq 0$, and in particular when $n=2$ or 3 . 
Define a bilinear form on the vector space $L(S)$ by

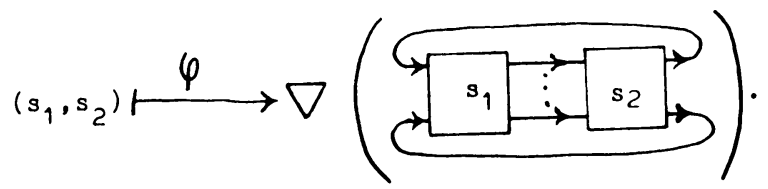

$\varphi$ clearly preserves skein moves and so extends over $L(S)$. Since $\left\{s_{i}^{*}\right\}$ is a basis of $L(S)^{*}$, there are elements $a_{k, l}$ of $F$ such that $\varphi=\Sigma a_{k, l} s_{k}^{*} \otimes s_{l}^{*}$. So

$$
\begin{aligned}
\varphi\left(s_{i}, s_{j}\right) & =\sum_{k, l} a_{k}^{*}\left(s_{i}\right) s_{l}^{*}\left(s_{j}\right)=\sum a_{k, l} M_{k, i} M_{l, j} \\
& =\sum_{l}\left(\sum_{k} a_{k, l} M_{k, i}\right) M_{l, j} \\
& =\left(\left(A^{T} M\right)^{T} M\right)_{i, j}=\left(M^{T} A M\right)_{i, j},
\end{aligned}
$$

where $M=M_{n}$ and $(A)_{k, l}=a_{k, l}$. But

$$
\varphi\left(s_{i}, s_{j}\right)=\nabla\left(s_{i} s_{j}\right)=M_{i, j}
$$

and so $M=M^{T} A M$, i.e., $\varphi=A=\left(M^{T}\right)^{-1}$. So if $\alpha=\Sigma a_{i} s_{i}$ and $\beta=\Sigma b_{i} s_{i}$, we have

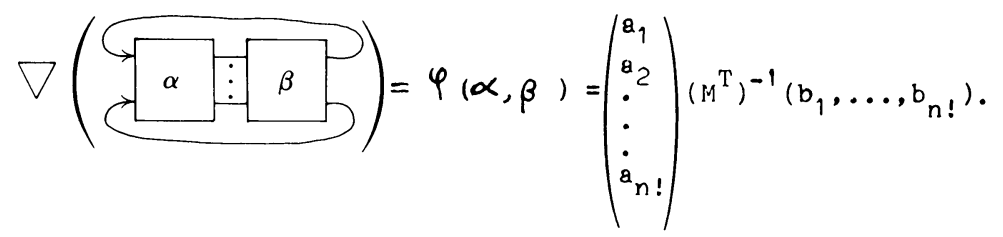

Special case. $n=2$. As computed above, using the basis

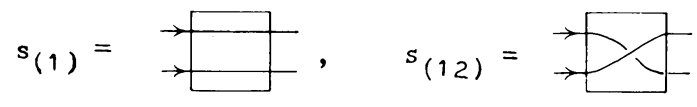

we find $M_{2}=\left(\begin{array}{ll}0 & 1 \\ 1 & z\end{array}\right)$ so that

$$
A=\left(M_{2}^{T}\right)^{-1}=\left(\begin{array}{cc}
-z & 1 \\
1 & 0
\end{array}\right)
$$

Thus $\varphi(a, b)=\left(-z s_{(1)}^{*} \otimes s_{(1)}^{*}+s_{(1)}^{*} \otimes s_{(12)}^{*}+s_{(12)}^{*} \otimes s_{(1)}^{*}\right)(a, b)$. We claimed before that similar formulas could be derived for any room. As an example, let us make similar computations for the room

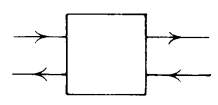


92

C. A. GILDER

Let $s_{1}$ and $s_{2}$ be two skein elements of this room; we calculate the bilinear form $\psi$ given by

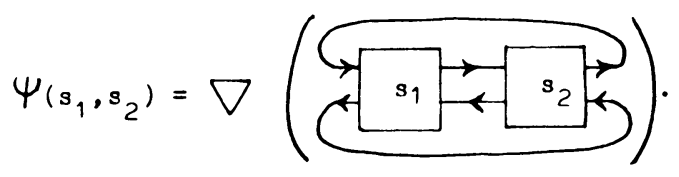

First define skein elements of our previous room:
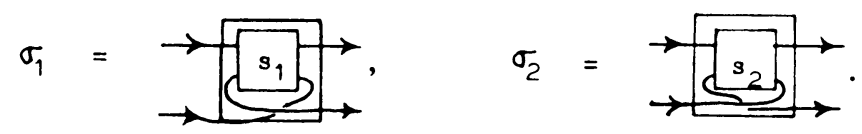

One sees easily that $\psi\left(s_{1}, s_{2}\right)=\varphi\left(\sigma_{1}, \sigma_{2}\right)$. Following Conway, we define

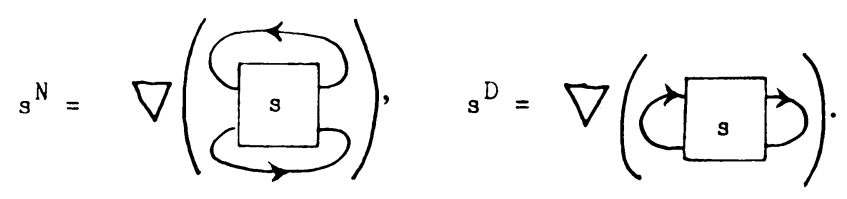

As before, $N$ and $D$ define dual elements for this room ( $N$ and $D$ stand for numerator and denominator respectively. This notation will become less mysterious shortly).

By drawing links one sees that
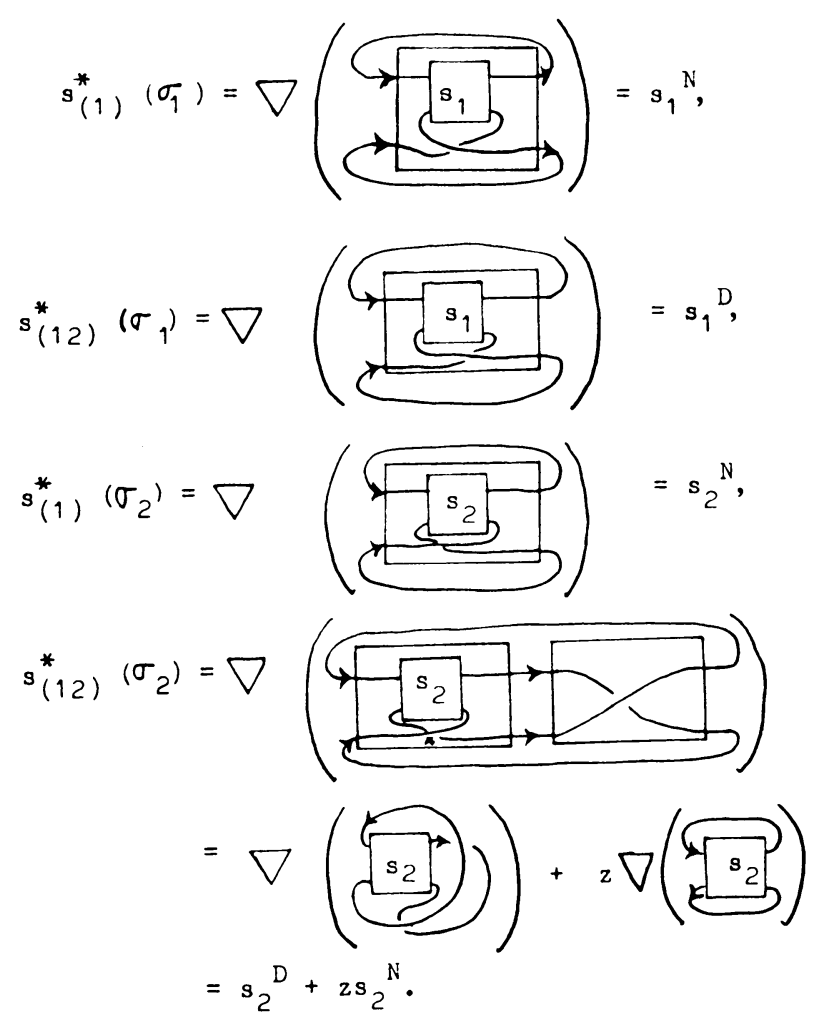
Then by the formula for $\varphi$ above,

$$
\begin{aligned}
\psi\left(s_{1}, s_{2}\right) & =\varphi\left(\sigma_{1}, \sigma_{2}\right)=-z s_{(1)}^{*}\left(\sigma_{1}\right) s_{(1)}^{*}\left(\sigma_{2}\right)+s_{(1)}^{*}\left(\sigma_{1}\right) s_{(12)}^{*}\left(\sigma_{2}\right)+s_{(12)}^{*}\left(\sigma_{1}\right) s_{(1)}^{*}\left(\sigma_{2}\right) \\
& =-z s_{1}^{N} s_{2}^{N}+s_{1}^{N}\left(s_{2}^{D}+z s_{2}^{N}\right)+s_{1}^{D} s_{2}^{N} \\
& =s_{1}^{N} s_{2}^{D}+s_{1}^{D} s_{2}^{N} .
\end{aligned}
$$

Similarly, using the bilinear form defined by

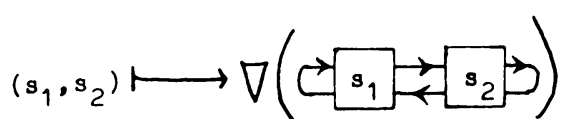

we get

$$
\nabla\left(C s_{1}-s_{2} D\right)=s_{1}^{D} s_{2}^{D} .
$$

Furthermore, note that a basis for the linearization of this room can be taken to be two elements which Conway calls 0 and $\infty$ :
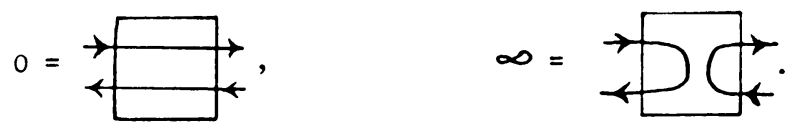

Note that

$$
0^{N}=0, \quad \infty^{N}=1, \quad 0^{D}=1, \quad \infty^{D}=0 .
$$

We continue to abuse notation and write $s^{N}$ and $s^{D}$ for the links

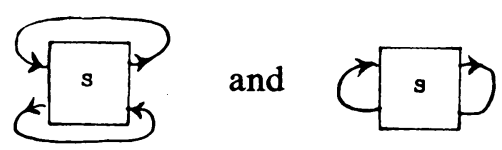

respectively. We also write $s_{1}+s_{2}$ for the skein element

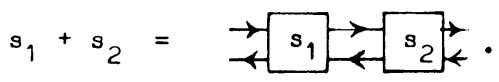

Summarizing,

Proposition 15.

$$
\begin{aligned}
& \left(s_{1}+s_{2}\right)^{N}=\nabla\left(\underset{s}{s_{1} \rightarrow s_{2} \rightarrow}\right)=s_{1}^{N} s_{2}^{D}+s_{1}^{D} s_{2}^{N}, \\
& \left(s_{1}+s_{2}\right)^{D}=\nabla\left(s_{1} B\right)=s_{1}^{D} s_{2}^{D},
\end{aligned}
$$

$0^{N}=0, \infty^{N}=1,0^{D}=1, \infty^{D}=0$.

The motivation for Conway's notation is now evident.

REMARK. We may summarize the polynomials of a skein element $s$ by writing the fraction $s^{N} / s^{D}$, so that Proposition 15 allows us to calculate polynomials using arithmetic for fractions. However, we may not cancel common terms from numerator and denominator. 
Definition. Let $L_{1}$ and $L_{2}$ be links in $S^{3}$ separated by an embedded $S^{2}$. A connected sum $L_{1} \# L_{2}$ is a link obtained from $L_{1} \cup L_{2}$ by connect summing a component of $L_{1}$ with a component of $L_{2}$. Of course, there may be more than one choice of $L_{1} \# L_{2}$.

COROllary 16. Let $L_{1}$ and $L_{2}$ be links. Then for any connected sum $L_{1} \# L_{2}$,

$$
\nabla\left(L_{1} \sharp L_{2}\right)=\nabla_{L_{1}} \nabla_{L_{2}} \text {. }
$$

(Compare Corollary 11.)

Proof. Let $K_{i}$ be the component of $L_{i}$ used to form the connected sum, let $a_{i}$ be an $\operatorname{arc}$ on $K_{i}$, and let $b_{i}$ be any arc of $L_{i}$. Let $s_{1}$ and $s_{2}$ be the skein elements obtained from $L_{1}$ and $L_{2}$ by deleting the arcs $a_{i}$ and $b_{i}$ so that the endpoints of $a_{1}$ appear to the right and those of $a_{2}$ appear to the left (see Figure 15). (If necessary, replace the arcs $\rightarrow$ by 30 to make the orientations match as shown.)
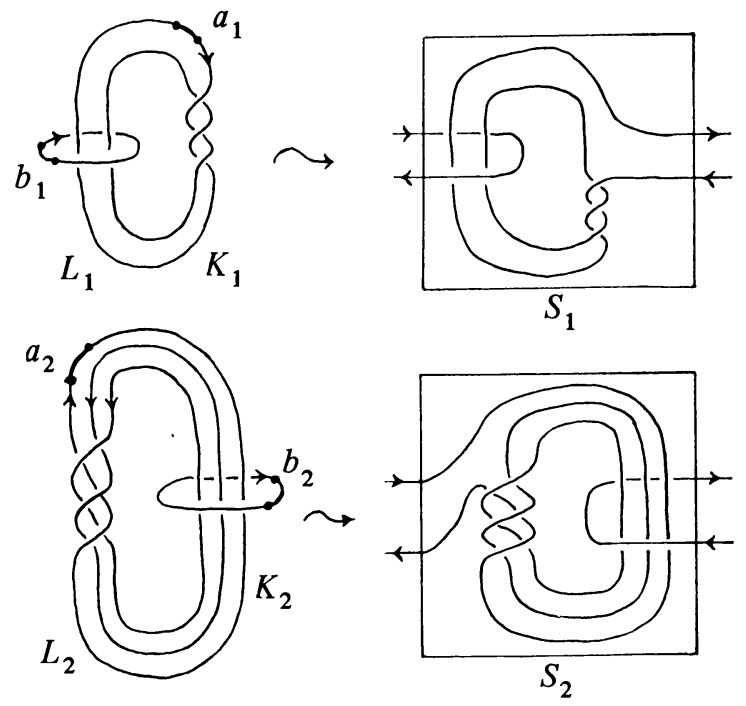

FIGURE 15

Then $s_{i}^{D}=L_{i}$, and

$$
\begin{aligned}
\nabla\left(L_{1} \# L_{2}\right) & =\left(s_{1}+s_{2}\right)^{D}=s_{1}^{D} s_{2}^{D} \quad \text { (by Proposition 15) } \\
& =\nabla_{L_{1}} \nabla_{L_{2}} .
\end{aligned}
$$

REMARK. Viro [V] points out that the various connected sums of two links are all mutations of one another in the sense of $\S 8$ of this paper, and so have identical polynomials.

Application. Let $s$ be a skein element of the room indicated in Figure 16, and let $L$ be the lift of $L_{0}$ below to the $p$-fold cover of $S^{3}$ branched along the unknotted component. We can write $L$ as a series of $p$ blocks as in Figure 17 and so $\nabla_{L}=(s+s+\ldots+s)^{N}=p s^{N}\left(s^{D}\right)^{p-1}$ by Proposition 15 and induction. 


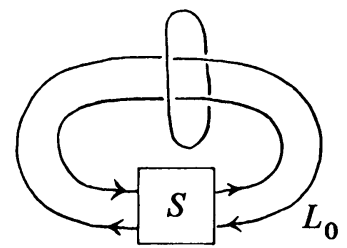

FIGURE 16

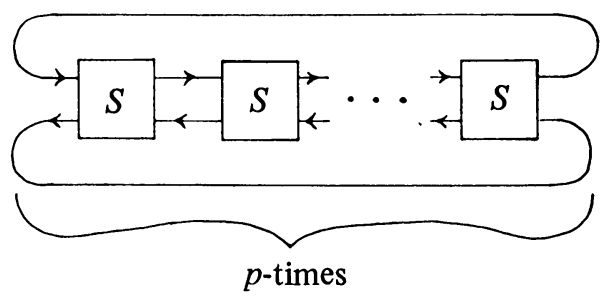

FIGURE 17

6. More examples. In the following examples, $a$ and $b$ are integers such that

$$
a+b=1, \quad b=2 k \geqslant 4 \text {. }
$$

Recall that 团 stands for $n$ half-twists, and that we will abuse notation as in §3. An asterisk $(*)$ by a crossing will indicate that a skein move will be performed at that crossing. Note that InI is equivalent to tation chosen will be such that arrows lead into the top of any box labelled $b$ and out of that labelled $a$; the consistency of this scheme is ensured in these examples by $b$ being even and $a$ being odd.

(a)

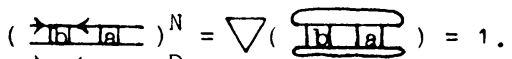

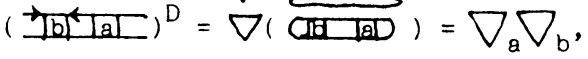

(b)

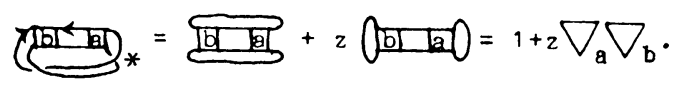

(c)
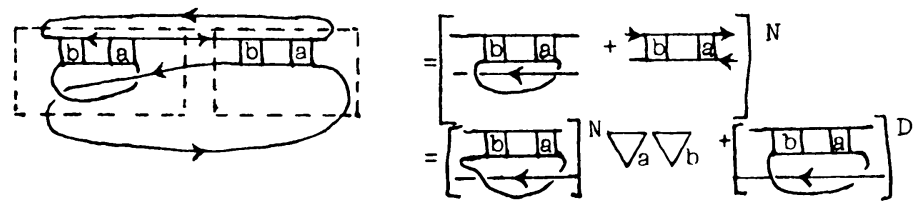

by Corollary 16. 
(by Proposition 15 and Corollary 16)

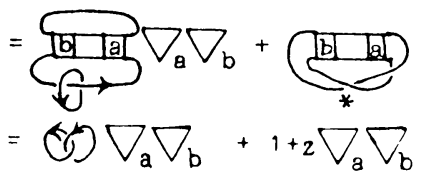

(by Example 6(b)) $=2 z \nabla_{a} \nabla_{b}+1$ (by Example 3(b)).

(d)

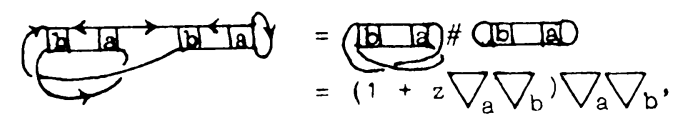

by Corollary 16 and the above examples.

(e)

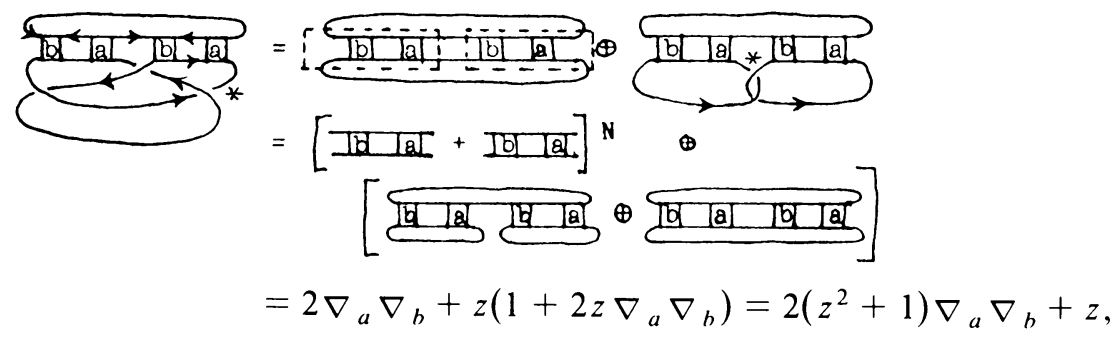

by Proposition 15 and the above examples; note that the second link is the unknot and that the third link is the first.

(f)

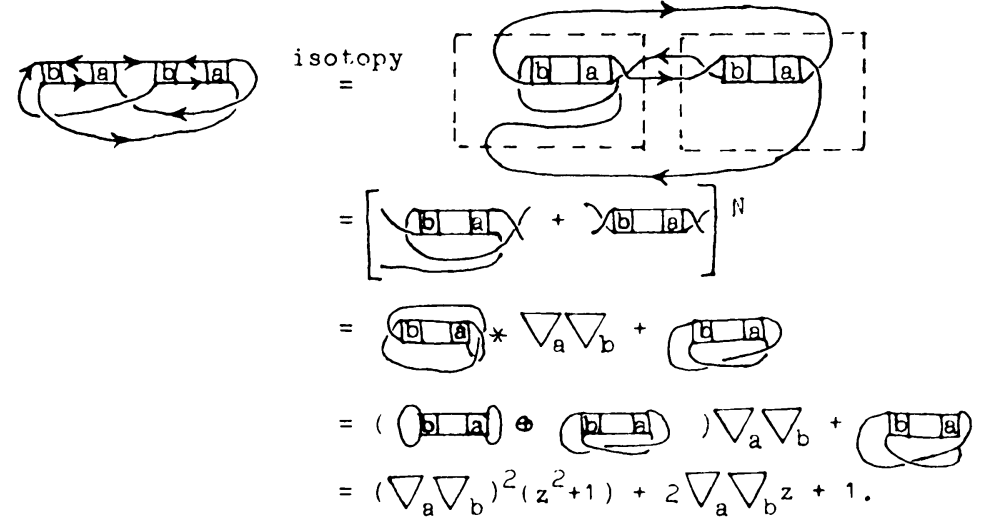

7. Another formula, 2-bridge knots, and signatures. This section is composed of three remarks.

REMARK 1. A similar argument as in $\$ 5$ using the room

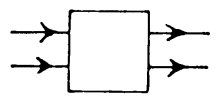

gives the formula $\nabla_{n+m}=\nabla_{n+1} \nabla_{m}+\nabla_{n} \nabla_{m+1}+z \nabla_{n} \nabla_{m}$. 


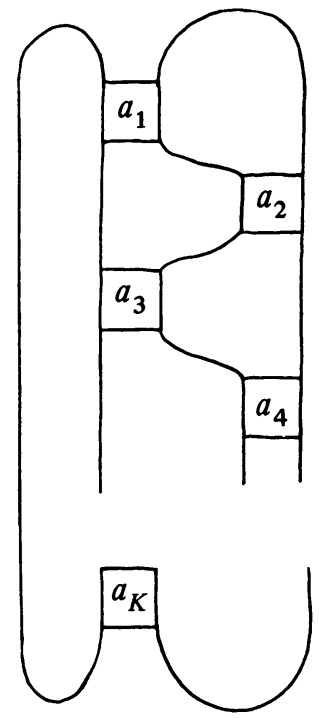

FIGURE 18

Remark 2. A 2-bridge knot is a knot of the form shown in Figure 18. Form the continued fraction

$$
\begin{aligned}
& p / q=\left[-a_{k}, a_{k-1},-a_{k-2}, \ldots,-a_{1}\right] \\
&\left(=-a_{k}+\frac{1}{a_{k-1}+\frac{1}{-a_{k-2}+\cdots}}\right) .
\end{aligned}
$$

Then it is well known [S] that two such knots giving either $p / q$ or $p /(p-q)$ are isotopic in $S^{3}$. The following is not hard to prove by induction.

Fact. Replacing $q$ by $p-q$ if necessary so that we may assume that $q$ is even, $p / q$ has a unique continued fraction expansion with even coefficients, $p / q=$ $\left[2 b_{1}, 2 b_{2}, \ldots, 2 b_{2 n}\right]$.

The polynomials of these knots are given by

THEOREM 17 (CONWAY). $\nabla$ of the above knot is the numerator of $\left[-z b_{1}, z b_{2}\right.$, $\left.-z b_{3}, \ldots, z b_{2 n}\right]$ expressed as a simple fraction, where the $b_{i}$ 's are as above.

SKETCH OF PROOF. By the fact above, we may assume $q$ is even, so

$$
p / q=\left[2 b_{1}, 2 b_{2}, \ldots, 2 b_{2 n}\right]=\left[2 b_{1}, 2 b_{2}, \ldots, 2 b_{2 n-1}-1,1,-\left(2 b_{2 n}+1\right)\right] .
$$

This last expansion of $p / q$ has an odd number of coefficients and so corresponds to a 2-bridge knot, all of whose $a_{i}$ 's are even except the last three. One may now prove the theorem for these types of knots using Proposition 15 and induction.

REMARK 3. The signature of $K, \sigma(K)$, may be calculated recursively with the following formulas:

$$
\begin{gathered}
\sigma\left(K_{l}\right) \leqslant \sigma\left(K_{r}\right) \leqslant \sigma\left(K_{l}\right)+2, \\
\operatorname{sign}\left(\nabla_{K}(2 i)\right)=i^{\sigma(K)},
\end{gathered}
$$


that is,

$$
\operatorname{sign}\left(\nabla_{K}(2 i)\right)=\operatorname{sign}\left(\Delta_{K}(-1)\right)= \begin{cases}1, & \text { if } \sigma(K) \equiv 0(\bmod 4), \\ -1, & \text { otherwise }\end{cases}
$$

where $K_{l}$ and $K_{r}$ are as in previous sections.

Formula (9a) follows from a simple consideration of the Seifert matrices of $K_{l}$ and $K_{r}$ as in $\S 1$; (9b) follows from [M3, p. 400].

To calculate $\sigma(K)$, we use $(9 \mathrm{~b})$ to determine $\sigma(K) \bmod 4$, and $(9 \mathrm{a})$ along with a record of the $\nabla$ 's to finish the calculation.

EXAMPLE. Let

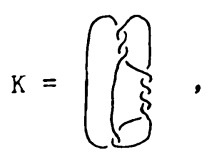

so that $p / q=[2,-4,-3]=23 / 13$. Replacing 13 by $23-13=10$, we write

$$
23 / 10=[2,4,-2,2]=[2(1), 2(2), 2(-1), 2(1)]
$$

so, by Theorem $17, \nabla_{K}=$ the numerator of $[-z, 2 z, z, z]=-2 z^{4}-2 z^{2}+1$. Alternatively, we can do skein moves to calculate $\nabla_{K}$ :

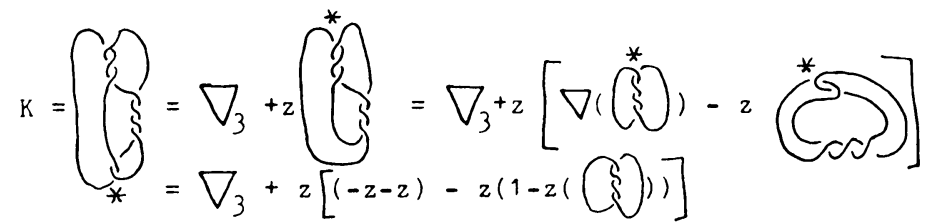

$$
\begin{aligned}
& =\nabla_{3}+z[(-2 z)-z(1+z(2 z))] \\
& =-2 z^{4}-2 z^{2}+1 \text {, as before. }
\end{aligned}
$$

So, changing a crossing of $K$ gives the knot

$$
\mathrm{K}_{3}=\sqrt{3}
$$

and changing a crossing of this knot gives the unknot; the corresponding $\nabla$ 's are $-2 z^{4}-2 z^{2}+1, z^{2}+1$, and 1 . We use (9) to compute signatures: $\operatorname{sign}\left(\nabla_{K_{3}}(2 i)\right)=$ -1 , and so by $(9 \mathrm{~b}), \sigma\left(K_{3}\right) \equiv 2(\bmod 4)$. Similarly, $\sigma(K) \equiv 2(\bmod 4)$. But by $(9 \mathrm{a})$, the signature can go up by at most 2 at each stage, so that $\sigma\left(K_{3}\right)=2=\sigma(K)$.

8. Mutations, skein equivalence, and the Conway-Kinoshita-Terasaka knots. Given a link $L$, we have seen that we can change $L$ to unlinks by a series of skein moves; we write $L$ as $L_{1} \oplus L_{2}$ or $L_{1} \ominus L_{2}$ and continue the process. We can associate a binary tree $T(L)$ to $L$ by drawing a node for each link in the skein decomposition, drawing an edge between the nodes corresponding to $L$ and $L_{1}$ and to $L$ and $L_{2}$, and writing $\mathrm{a}+$ or - at each node depending if a $\oplus$ or $\ominus$ occurred in the decomposition.

EXAMPle. Let

$$
K=(S)=O \oplus 00.0000 .
$$


So $T(K)$ is formed from

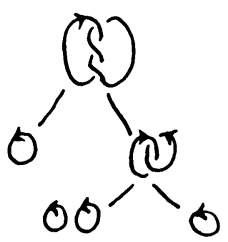

i.e.,

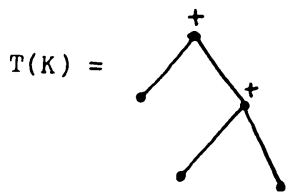

Note that $T(L)$ is by no means unique. For example, since

$$
L=L \oplus(L \cup O)
$$

(see §3a), we can add a branch to any bottom-most node of the tree:

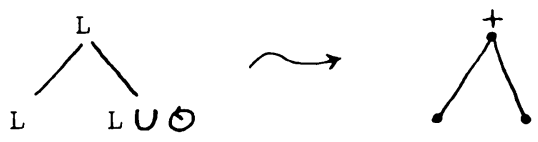

Hence we assume that all trees for the same link $L$ have an identical number $n(T)$ of node levels along any branch; the above tree for the trefoil has 3:

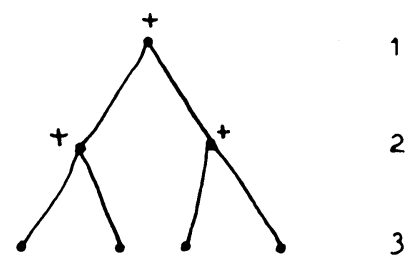

Labelling the nodes at the bottom level of a tree for $L$ as $n_{1}(L), \ldots, n_{k}(L)$, we write $\bar{n}_{i}(L)$ for the link represented by the node $n_{i}(L)$.

Definition. The two links $L_{1}$ and $L_{2}$ are said to be skein equivalent if there are isomorphic trees for $L_{1}$ and $L_{2}$ such that, for all $i$,

$$
\bar{n}_{i}\left(L_{1}\right)=\bar{n}_{i}\left(L_{2}\right)
$$

where $=$ means isotopic. As we have seen, skein equivalent links have the same Conway polynomials and signatures.

DEFinition. Suppose the following room appears in a link $L$ :

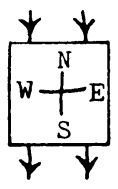


Define a mutation $m(L)$ of $L$ to be the link obtained from $L$ by rotating this room by $180^{\circ}$ :

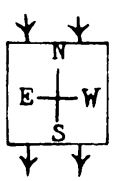

(so the orientation of the strands inside the room are preserved).

REMARK. We could carry out a similar program by rotating in the other direction.

Proposition 18 (CONWAY). L and $m(L)$ are skein equivalent.

PROOF. As proved in $\$ 5$, any skein element can be written as a formula in terms of $s_{(1)}, s_{(12)}, \oplus$ and $\Theta$. But mutation preserves the orientation of each strand, and hence preserves $\oplus$ and $\ominus$; mutation also clearly preserves $s_{(1)}$ and $s_{(12)}$. Thus by first writing the interiors of the rooms for $L$ and $m(L)$ in terms of $s_{(1)}$ and $s_{(12)}$ and then decomposing the outside of the room by further skein moves, we see that $L$ and $m(L)$ have trees with identical bottom row nodes.

The agreement of $\nabla$ and $\sigma$ for skein equivalent links also has a geometric reason:

Proposition 19 (Montesinos AND Viro). The 2-fold covers $M_{L}$ and $M_{m(L)}$ of $S^{3}$ branched along $L$ and $m(L)$ respectively are homeomorphic.
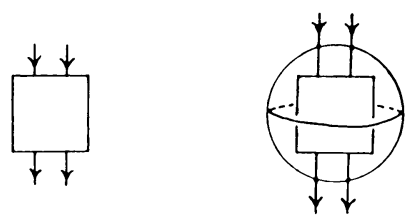

Figure 19

Proof. Let $B^{3}$ be a small ball containing the room as shown in Figure 19. Then $S^{3}=B \cup\left(S^{3}-B\right)$, and letting $\hat{A}$ be the 2-fold branched cover of $A \subseteq S^{3}$ branched along $A \cap L$, we have

$$
M_{L}=\hat{B} \underset{\partial \hat{B}=\hat{S}^{2}}{\cup} \widehat{S^{3}-B} .
$$

But $S^{2} \cap L$ consists of four points, and we can construct $\hat{S}^{2}$ as follows. The 2-fold branched cover of a disk along two points $a$ and $b$ is an annulus obtained by gluing together two copies of the disk cut along an arc connecting $a$ and $b$ (see Figure 20). Gluing two of these annuli together along their boundaries gives a torus as the 2-fold cover of $S^{2}$ branched on four points, i.e., $\hat{S}^{2}$.

But now the effect of the mutation is to rotate $B$ by $180^{\circ}$, so that the lift of this rotation to $S^{2}$ is a homeomorphism of the torus isotopic to the identity. Thus

$$
M_{m(L)}=\hat{B} \underset{h\left(S^{2}\right)}{\cup} \widehat{\left(S^{3}-B\right)}
$$

and so is homeomorphic to $M_{L}$. 


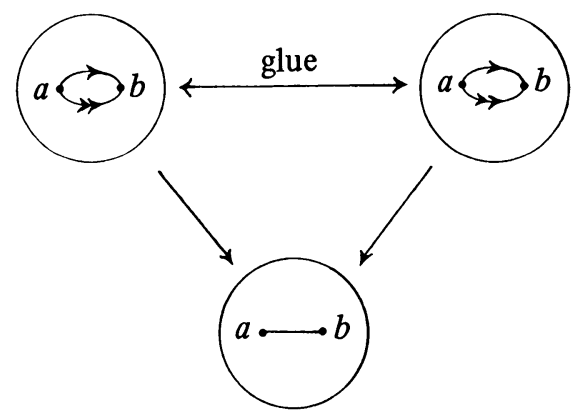

FIGURE 20

ExAmple. The following distinct knots ( $C$ for Conway and $K T$ for KinoshitaTerasaka [KT]) are skein equivalent; in fact they are mutants of each other via the indicated room (see Figure 21).

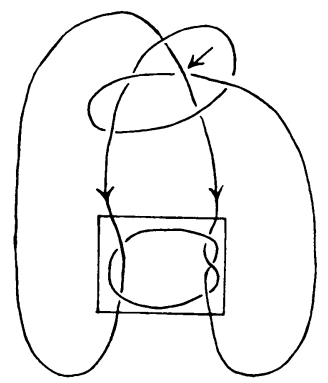

$C$

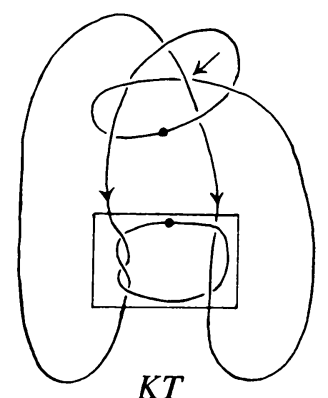

$K T$

\section{FIGURE 21}

Remarks. These knots can be seen to be distinct by examining representations of their fundamental groups [Ri]. They both have Conway polynomial 1 (in fact, they are the only 11 crossing knots with trivial Alexander polynomial) and both have unknotting number 1. $K T$ is known to be ribbon (the one ribbon move necessary is made by attaching a trivial band between the points on the knot indicated by dots), but $C$ is not known to be slice. The proposition above shows that the 2-fold branched covers of $C$ and $K T$ are homeomorphic (see also [V]), while [M] shows that these covers have nontrivial $\pi_{1}$.

Using the notation in $[\mathbf{R}]$, the $p$-fold branched covers of $C$ and $K T$ can be drawn as in Figure 20 where the framings on all components are -1 . For $p=2$, these pictures are identical as expected (see also $[\mathbf{M}])$. I do not know if they represent the same 3-manifolds for $p>2$; in fact, since $\nabla(C)=\nabla(K T)=1$, they are all homology $S^{3}$ s. Note however that a rotation about the indicated $S^{2}$ in the drawing for the cover of $C$ gives that for $K T$; this $S^{2}$ is simply the lift of the original $S^{2}$ describing the mutation from $C$ to $K T$. 


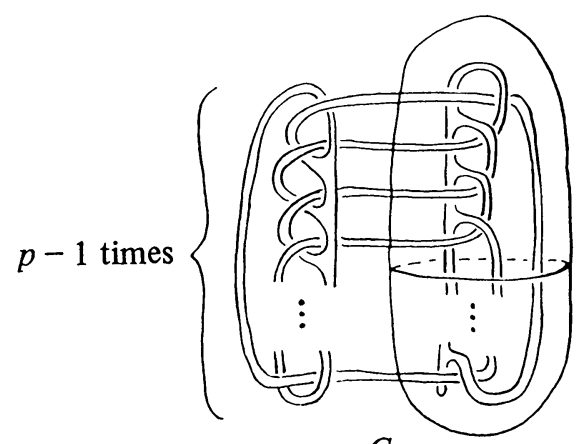

C

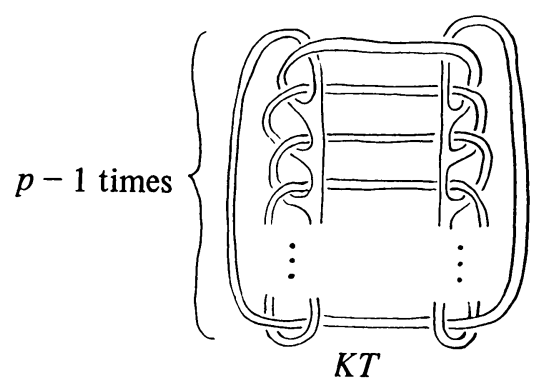

FIGURE 22

REMARK. These pictures were obtained using methods in [R]. The knots were unknotted at the crossings indicated by arrows by blowing up an unknotted component $P$ of framing -1, drawing the cover of the newly formed unknot, and keeping track of $P$.

III. The example. The following asymmetric link of two unknotted components $K_{1}$ and $K_{2}$ with linking number +1 satisfies the distinct lifting property for all $p \geqslant 2$ (see Figure 23).

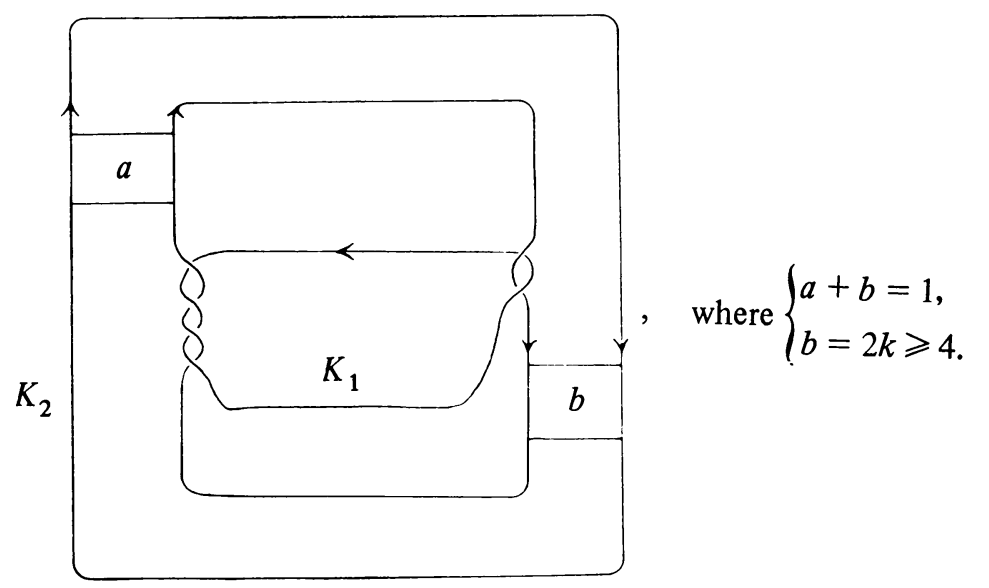

FIGURE 23 
Remarks. (1) The link shown in Figure 24 is an example of such a family with linking number zero.

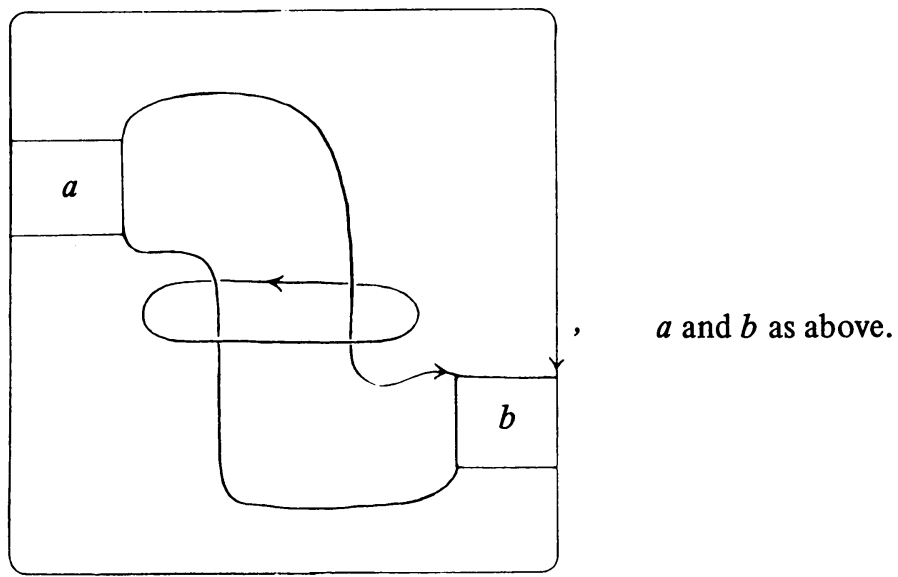

\section{FIGURE 24}

(2) The case of linking number one is of more interest here because the associated lifts are in fact knots.

Let $\hat{K}_{i}$ be the lift of $K_{i}$ to the $p$-fold cover of $K_{j}, j \neq i$. We shall distinguish $\hat{K}_{1}$ from $\hat{K}_{2}$ by their potential functions, and in particular by showing degree $\nabla \hat{K}_{1}<$ degree $\nabla \hat{K}_{2}$.

Proposition 20. degree $\nabla_{K_{1}} \leqslant 2 p$.

Proof. Consider the punctured torus $T$ which $K_{1}$ bounds. Note that $K_{2}$ intersects $T$ in one point (see Figure 25).

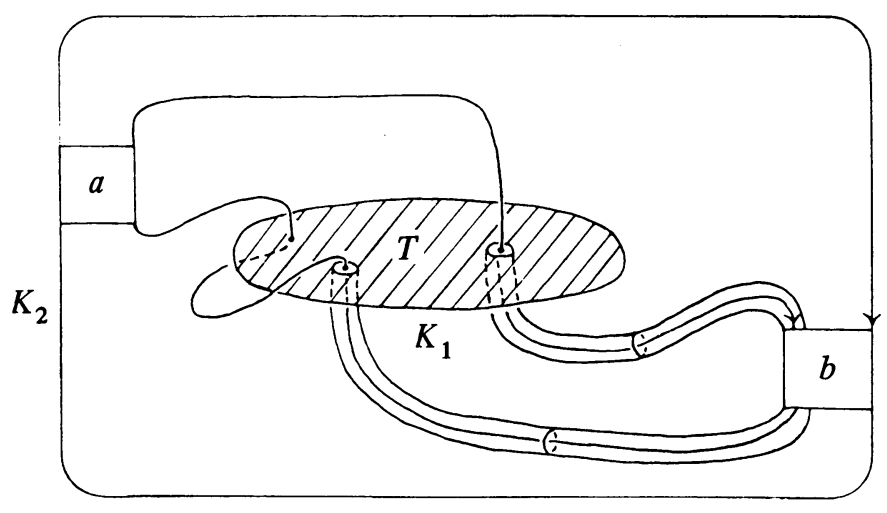

Figure 25

Since the linking number of the two components is $1, \hat{K}_{1}$ has one component, and so the lift $\hat{T}$ of $T$ to the $p$-fold cover of $K_{2}$ is a connected $p$-fold cover of $T$ branched on one point. Thus

$$
\chi(\hat{T})=p \chi(T)-(p-1)=1-2 p
$$


and so the genus of $\hat{T}$ is $p$. But $\hat{T}$ is a Seifert surface for $\hat{K}_{1}$, and so the degree of $\Delta_{K_{1}}(t)$ as a polynomial in $t$ with constant coefficient is $\leqslant 2 p$, i.e., by (6),

$$
\text { degree } \nabla_{\hat{K}_{1}} \leqslant 2 p \text {. }
$$

THEOREM 21. degree $\nabla \hat{K}_{2}=2(p-1)(b-1)$.

COROLlaRY 22. degree $\nabla \hat{K}_{2}>$ degree $\nabla_{\hat{K}_{1}}$ for $b \geqslant 4, p \geqslant 2$.

Proof. The corollary follows immediately from the theorem and from (10). To prove the theorem, we first draw $\hat{K}_{2}$ and then use Conway's calculus to calculate its $\nabla$. Cutting along the obvious disk bounded by $K_{1}$, we obtain
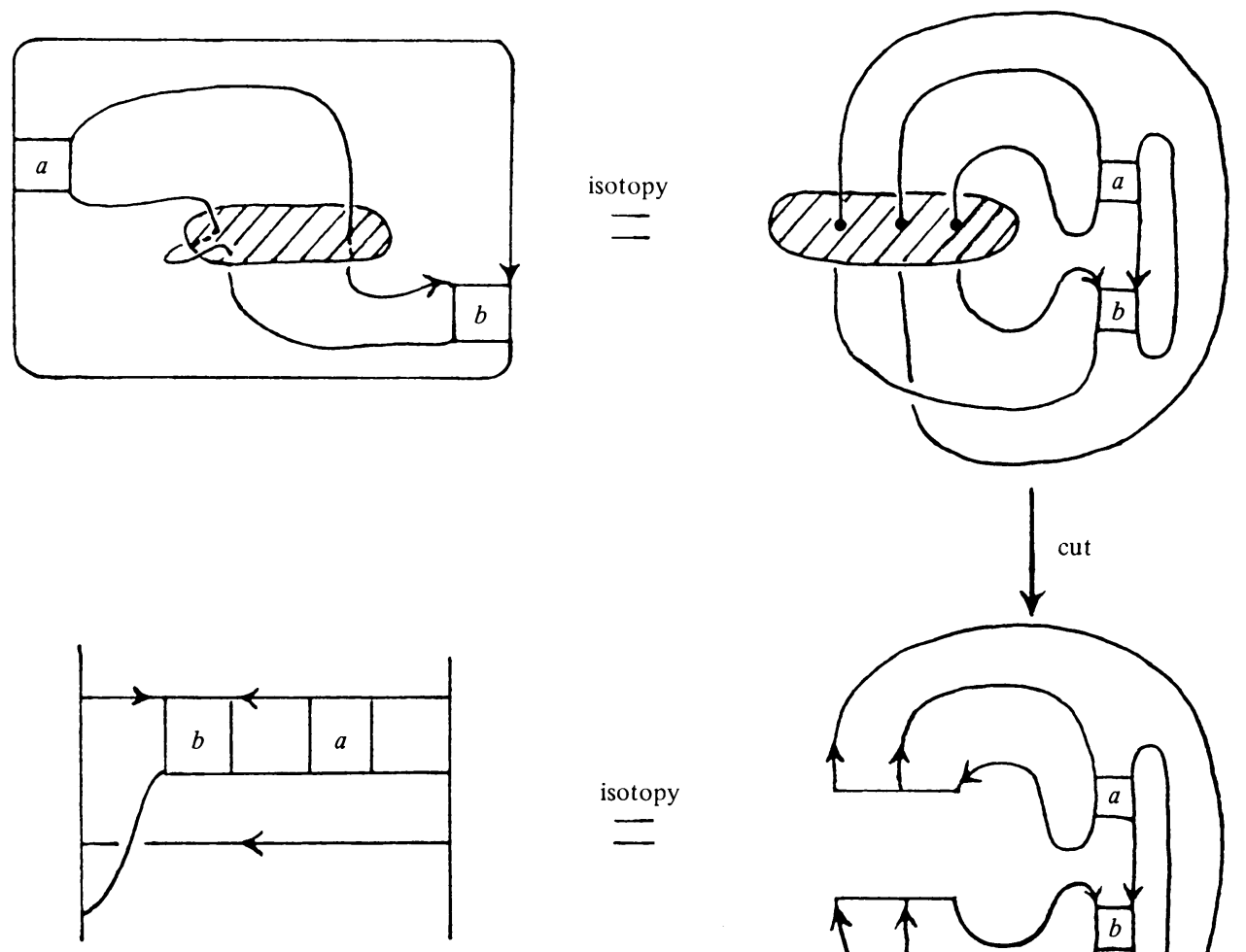

isotopy

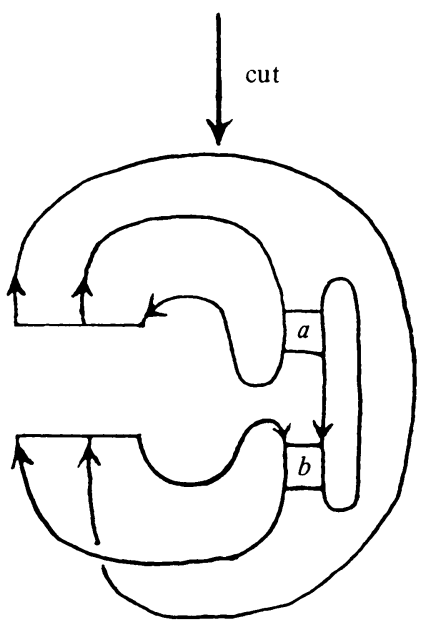

FIGURE 26

To draw $\hat{K}_{2}$ we place $p$ of these blocks end-to-end and connect:

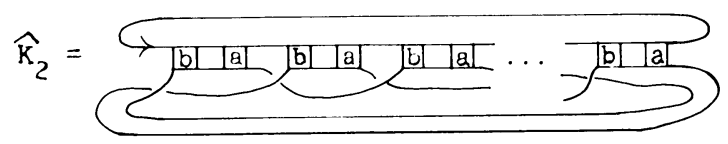


We can now use room theory to calculate $\nabla \hat{K}_{2}$ inductively. First let

$$
s_{n}=\text { bl }
$$

When convenient, we will write this room as

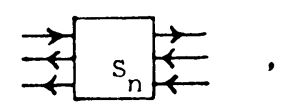

so that

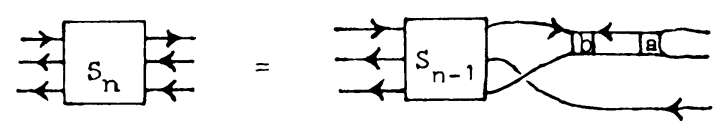

Further, define skein elements $S_{i, n}$ by

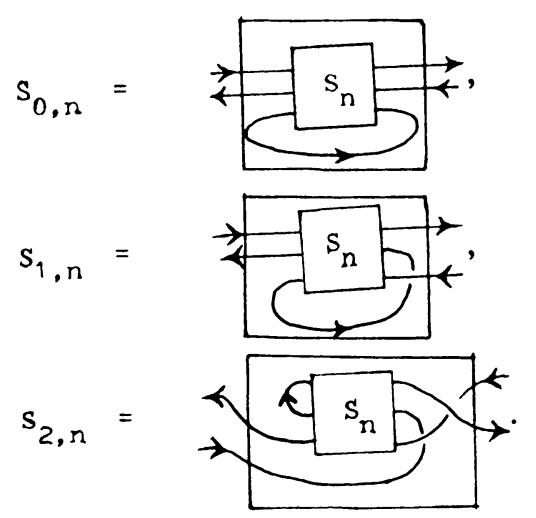

We shall follow two conventions. First, since the orientations always lead to arrows pointing into the top of the box labeled $b$ and out of that labeled $a$, we will not always draw them in the pictures. Second, we will use an asterisk (*) as before to indicate a skein move.

From the picture of $\hat{K}_{2}$ on the previous page, we see that we desire $S_{0, p}^{N}$. But by (11),

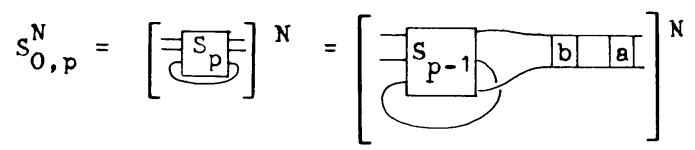

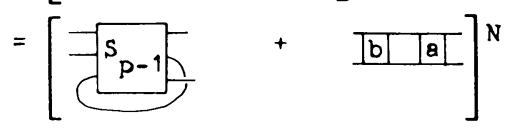

$$
\begin{aligned}
& =\left[\mathrm{s}_{1, \mathrm{p}-1}+\mathrm{b}|\mathrm{b}|\right]^{N} \text {. }
\end{aligned}
$$

To use Proposition 15 for $(A+B)^{N}$, we need to know $A^{N}, B^{N}, A^{D}$, and $B^{D}$. Thus we must compute $S_{1, n}^{N}$ and $S_{1, n}^{D}$. 
106

C. A. GILLER

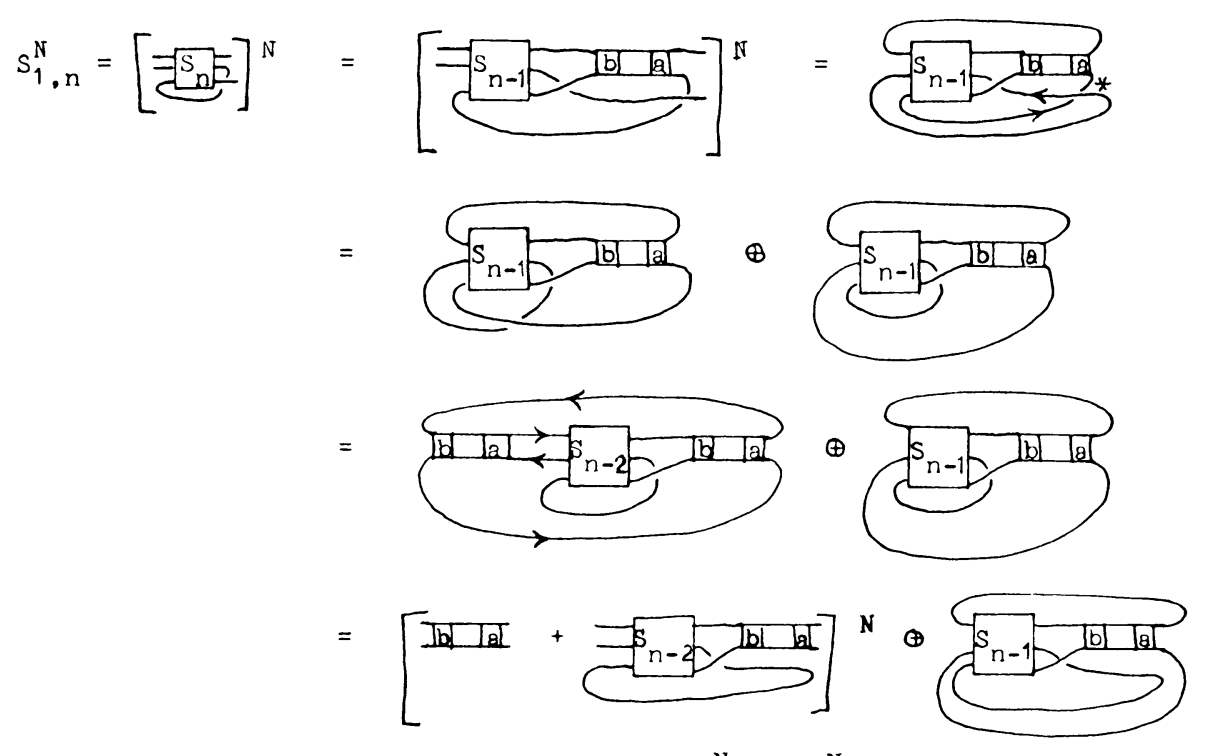

$=\left(\text { 且国 }+s_{0, n-1}\right)^{N} \oplus s_{0, n}^{N}$.
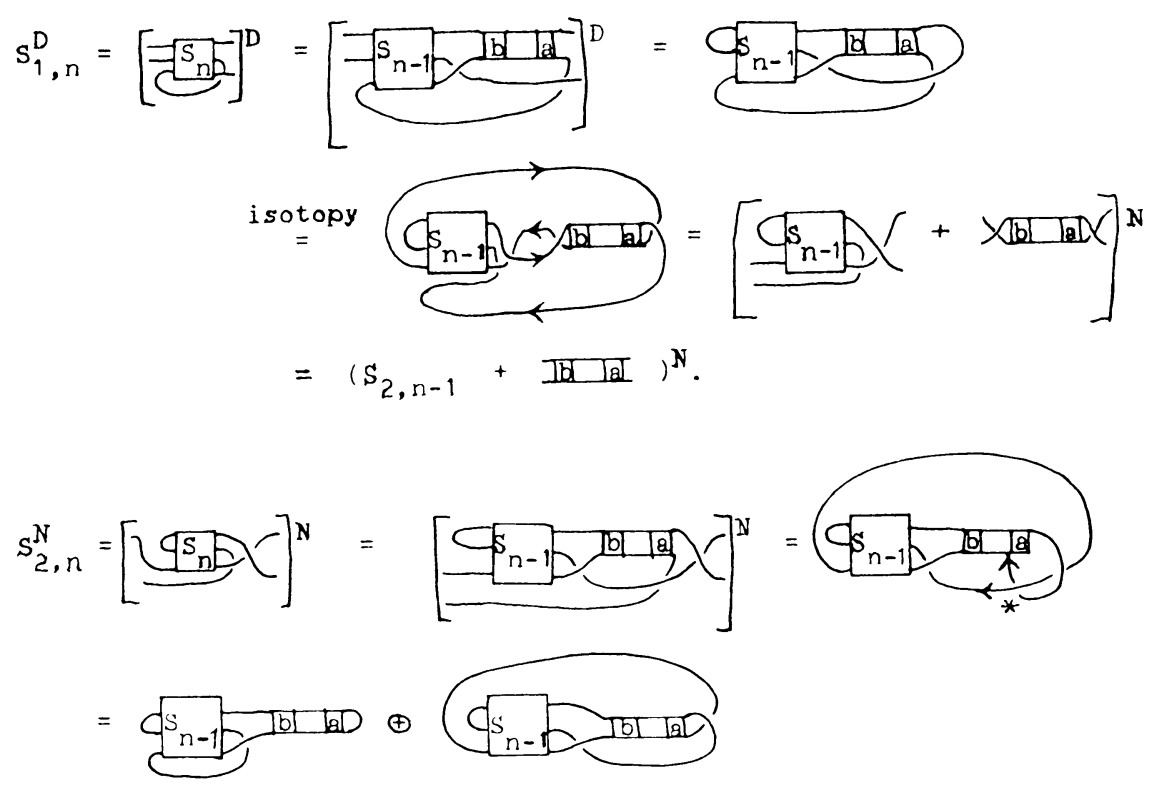

$=s_{1, n-1}^{D} \#($ G

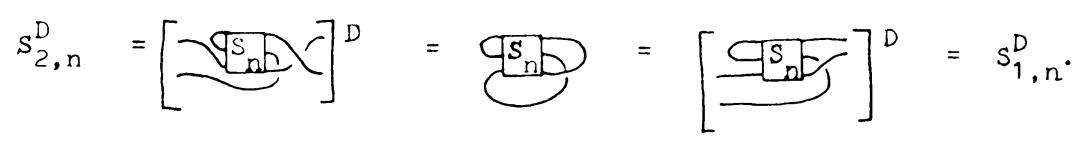


And finally,

$$
s_{0, n}^{D}=\left[\pi s_{n}\right]^{D}=s_{n-1}
$$

Summarizing,

$$
\begin{aligned}
& s_{0, n}^{N}=\left(s_{1, n-1}+\text { IDI Ial }\right)^{N}, \\
& s_{0, n}^{D}=s_{1, n-1} \#(\text { b }|a|) \text {, } \\
& s_{1, n}^{N}=\left(\text { IDI }+s_{0, n-1}\right)^{N} \oplus s_{0, n}^{N} \text {, } \\
& s_{1, n}^{D}=\left(s_{2, n-1}+\text { I I I I II }\right)^{N} \text {, } \\
& s_{2, n}^{N}=\left(s_{1, n-1}^{D} \# \text { b d D }\right) \oplus S_{1, n}^{D} \text {, } \\
& s_{2, n}^{D}=s_{1, n}^{D} \text {. }
\end{aligned}
$$

Before evaluating $\nabla$ of these links, note that $a+b=1$ and so, by Lemma 9, $\nabla_{a}=\nabla_{1-b}=\nabla_{b-1}$. Furthermore, letting $Q=\nabla_{b} \nabla_{b-1}$, we see that Example 6(a) can be written as

$$
\begin{aligned}
& (\text { IbI IaI })^{D}=\nabla_{b} \nabla_{a}=\nabla_{b-1} \nabla_{b}=a, \\
& (\text { Ib|a| })^{N}=1 .
\end{aligned}
$$

Note that, by Lemma 9, degree $Q=2 b-3$.

So, using our formulas for $\oplus, \sharp,(A+B)^{N}$ and $(A+B)^{D}$, we can eliminate the $S_{2}$ 's and rewrite (16) as

$$
\begin{aligned}
S_{0, p}^{N} & =Q S_{1, p-1}^{N}+S_{1, p-1}^{D}, \quad S_{0, p}^{D}=Q S_{1, p-1}^{D}, \\
S_{1, p-1}^{N} & =S_{0, p-2}^{D}+Q S_{0, p-2}^{N}+z S_{0, p-1}^{N}, \\
S_{1, p-1} & =Q^{2} S_{1, p-3}^{D}+z Q S_{1, p-2}^{D}+S_{1, p-2}^{D} .
\end{aligned}
$$

The examples in $\S 6$ can also be written in terms of $Q$ :

$$
\begin{aligned}
& S_{0,2}^{N}=2 z Q+1, \\
& S_{0,2}^{D}=z Q^{2}+Q, \\
& S_{1,2}^{N}=2\left(z^{2}+1\right) Q+z, \\
& S_{1,2}^{D}=Q^{2}\left(1+z^{2}\right)+2 z Q+1, \\
& S_{1,1}^{D}=z Q+1 .
\end{aligned}
$$

We now use these recursion formulas to gain a formula for degree $\nabla \hat{K}_{2}$.

Proposition 23. Consider the polynomials in (14) as polynomials in $Q$ with coefficients in $\mathbf{Z}[z]$. Then for each of these polynomials,

(1) The term containing the highest power of $Q$ has coefficient containing the highest power of $z$; hence this term is of highest degree if considered as a polynomial in $z$ alone. 
(2) These distinguished terms are as follows:

\begin{tabular}{c|c} 
polynomial & term \\
\hline$S_{0, p}^{N}$ & $Q^{p-1}[p-1]$ \\
$S_{0, p}^{D}$ & $Q^{p}[p-1]$ \\
$S_{1, p}^{N}$ & $Q^{p-1}[p]$ \\
$S_{1, p}^{D}$ & $Q^{p}[p]$
\end{tabular}

where we are writing $[n]$ for a polynomial in $z$ of degree $n$ with nonnegative coefficients.

Proof. This is a straightforward induction using (14) and the examples in $§ 6$. The proposition is true for $p=2$ by the examples. We give details only for the case of $S_{0, p}^{N}$. Assume true for $k \leqslant p$; then by (14),

$$
\begin{aligned}
S_{0, p+1}^{N} & =Q S_{1, p}^{N}+S_{1, p}^{D} \\
& =Q\left(Q^{p-1}[p]\right)+Q^{p}[p]+\text { lower order terms } \\
& =Q^{p}[p]+\text { lower order terms }
\end{aligned}
$$

since the coefficients in $[p]$ are nonnegative.

Clearly, (1) and (2) are still satisfied by $S_{0, p+1}^{N}$. The other cases follow similarly.

So we finally have our

THEOREM 24. $\operatorname{deg} \nabla \hat{K}_{2}=\operatorname{deg} S_{0, p}^{N}=2(p-1)(b-1)$.

Proof. By Proposition 23,

$$
\begin{aligned}
\operatorname{deg} S_{0, p}^{N} & =p-1+\operatorname{deg} Q^{p-1} \\
& =p-1+(p-1)(2 b-3)=2(p-1)(b-1) .
\end{aligned}
$$

REMARKs. (1) As mentioned before, a similar link provides an example of such a family with linking number zero (the proof is entirely similar to that given above).

(2) Murasugi [M2] gives formulas for the polynomials and signatures of $\hat{K}_{1}$ and $\hat{K}_{2}$ in terms of those of the link; in our case, the signatures are identical.

(3) Drawing $\hat{K}_{1}$ is possible but messy.

We now show that, as promised, we can produce for each $p \geqslant 2$ an infinite family of pairs of distinct knots with homeomorphic $p$-fold covers.

LEMMA 25. The p-fold cyclic covers of $S^{3}$ branched along $\hat{K}_{1}$ and $\hat{K}_{2}$ are homeomorphic.

Proof. Let $M$ be the $p$-fold cover of $S^{3}-L$ corresponding to the map $\varphi$ :

$$
\pi_{1}\left(S^{3}-L\right) \stackrel{\pi}{\rightarrow} H_{1}\left(S^{3}-L\right)=\mathbf{Z}_{1} \oplus \mathbf{Z}_{2} \rightarrow \mathbf{Z}_{1} \rightarrow \mathbf{Z} / p
$$

where $\mathbf{Z}_{i} \cong \mathbf{Z}$. Then $\pi_{1}(M)=G \subseteq \pi_{1}\left(S^{3}-L\right)$ is the kernel of $\varphi$ and $M$ is the complement of $\hat{K}_{2}$ in the $p$-fold cyclic cover of $S^{3}-K_{1}$. 
Now, $\pi(G) \supseteq\left(0, \mathbf{Z}_{2}\right)$, so there is a homeomorphism $G \rightarrow \mathbf{Z}_{2}$. Let $\bar{M}$ be the $p$-fold cover of $M$ corresponding to the map $G \rightarrow \mathbf{Z}_{2} \stackrel{P_{2}}{\rightarrow} \mathbf{Z} / p$. Then $\bar{M}$ is a $p^{2}$-fold cover of $S^{3}-L$. For let $\pi_{1}(\bar{M})=\bar{G} \subseteq G$. We have a commutative diagram:

$$
\begin{array}{ccccccc}
G & \hookrightarrow & \pi_{1}\left(S^{3}-L\right) & \rightarrow & \mathbf{Z}_{1} \oplus \mathbf{Z}_{2} & \rightarrow & \mathbf{Z}_{1} \stackrel{P_{1}}{\rightarrow} \mathbf{Z} / p \\
\text { UI } & \multicolumn{1}{c}{} & & & & & \\
\bar{G} & \rightarrow & G & \rightarrow & \mathbf{Z}_{2} & \stackrel{P_{2}}{\rightarrow} & \mathbf{Z} / p
\end{array}
$$

Note that $\pi_{1}\left(S^{3}-L\right) / \bar{G} \cong \pi_{1}\left(S^{3}-L\right) / G \oplus G / \bar{G}=\mathbf{Z} / p \oplus \mathbf{Z} / p$, and that $\bar{M}$ is a cover of $S^{3}-L$ corresponding to the map

$$
\pi_{1}\left(S^{3}-L\right) \rightarrow H_{1}\left(S^{3}-L\right) \stackrel{P_{1} \oplus P_{2}}{\rightarrow} \mathbf{Z} / p \oplus \mathbf{Z} / p
$$

But the same map would be obtained had we started with the cover of $S^{3}-K_{2}$ and formed the corresponding $p^{2}$-fold cover of $S^{3}-L$.

\section{REFERENCES}

[C1] J. H. Conway, An enumeration of knots and links, and some of their algebraic properties, Computational Problems in Abstract Algebra (John Leech, ed.), Pergamon Press, Oxford and New York, 1969.

[C2] __ Talks at Cambridge Math. Conf. (Summer, 1979).

[I] I. M. Isaacs, Character theory of finite groups, Academic Press, New York, 1976.

[K] L. Kauffman, The Conway polynomial, Topology 20 (1981), 101-108.

[KT] S. Kinoshita and H. Terasaka, On unions of knots, Osaka Math. J. 9 (1957), 131-153.

[Kb] R. Kirby, Problems in low dimensional manifold theory, Proc. Sympos. Pure Math., vol. 32, part 2, Amer. Math. Soc., Providence, R. I., 1978, pp. 273-312.

[M] J. M. Montesinos, Surgery on links and double branched covers of $S^{3}$, Knots, Groups and 3-Manifolds (L. P. Neuwirth, ed.), Ann. of Math. Studies, no. 84, Princeton Univ. Press, Princeton, N. J., 1975.

[M1] K. Murasugi, On periodic knots, Comment. Math. Helv. 46 (1971), 162-174.

[M2] __ On the signature of links, Topology 9 (1970), 283-298.

[M3] _ On a certain numerical invariant of link types, Trans. Amer. Math. Soc. 117 (1965), $387-422$.

[Ri] R. Riley, Homomorphisms of knot groups on finite groups, Math. Comp. 25 (1971), 603-619.

[R] D. Rolfson, Knots and links, Publish or Perish, Berkeley, Calif., 1976.

[S] H. Schubert, Knoten mit Zwei Brücken, Math. Z. 65 (1956), 133-170.

[V] O. Ya. Viro, Nonprojecting isotopies and knots with homeomorphic coverings, J. Soviet Math. 12 (1979), 86-96.

Current address: Class of 1984, Office of Student Affairs, Center for the Health Sciences, UCLA School of Medicine, Los Angeles, California 90024 